\title{
Analysis of 3D problems using a new enhanced strain hexahedral element
}

\author{
P. M. A. Areias*,, J. M. A. César de Sá, C. A. Conceição António \\ and A. A. Fernandes \\ Instituto de Engenharia Mecânica (IDMEC), Faculdade de Engenharia da Universidade do Porto, \\ Rua Dr. Roberto Frias s/n, 4200-465 Porto, Portugal
}

\begin{abstract}
SUMMARY
The now classical enhanced strain technique, employed with success for more than 10 years in solid, both $2 \mathrm{D}$ and $3 \mathrm{D}$ and shell finite elements, is here explored in a versatile 3D low-order element which is identified as HIS. The quest for accurate results in a wide range of problems, from solid analysis including near-incompressibility to the analysis of locking-prone beam and shell bending problems leads to a general 3D element. This element, put here to test in various contexts, is found to be suitable in the analysis of both linear problems and general non-linear problems including finite strain plasticity. The formulation is based on the enrichment of the deformation gradient and approximations to the shape function material derivatives. Both the equilibrium equations and their variation are completely exposed and deduced, from which internal forces and consistent tangent stiffness follow. A stabilizing term is included, in a simple and natural form. Two sets of examples are detailed: the accuracy tests in the linear elastic regime and several finite strain tests. Some examples involve finite strain plasticity. In both sets the element behaves very well, as is illustrated in numerous examples. Copyright (c) 2003 John Wiley \& Sons, Ltd.
\end{abstract}

KEY WORDS: finite elements; hexahedral element; finite strains; enhanced strains; brick element

\section{INTRODUCTION}

The analysis of general solid mechanics problems from linear elastic beam bending to finite strain elasto-plastic problems can be accomplished with the use of solid 3D elements and, in particular, hexahedral elements. Objections to such a one-size fits all approach are related both to the cost of the analysis and its accuracy. In most commercial finite element codes, problems with beams or shells are analysed with special-purpose elements. In terms of cost of

\footnotetext{
${ }^{*}$ Correspondence to: P. M. A. Areias, Instituto de Engenharia Mecânica (IDMEC), Faculdade de Engenharia da Universidade do Porto, Rua Dr. Roberto Frias s/n, 4200-465 Porto, Portugal.

†E-mail: simplas2002@netscape.net

Contract/grant sponsor: Ministério de Ciência e da Tecnologia, FCT; contract/grant number: PRAXIS XXI/BD/ $18538 / 98$

Received 9 September 2002

Revised 12 February 2003

Copyright (c) 2003 John Wiley \& Sons, Ltd.

Accepted 14 March 2003
} 
the analysis, it is clear that a beam element results in faster analyses than a general purpose $3 \mathrm{D}$ element. For the solution of shell problems with shell elements, the comparison is not so conclusive. In terms of versatility, the so-called solid-shell elements [1-7] which only adopt displacement variables at the nodes and do not preclude the use of general 3D constitutive models, can be effective in modelling shell intersections and allow a natural coupling with 3D elements. However, for problems containing thick geometries such as bulk forming problems, solid elements should be used.

The inspection of the accuracy of solid elements in the analysis of shell structures was carried out in References [8-15] with interesting conclusions for sufficiently refined meshes.

This paper presents a new stabilized enhanced strain 3D element which contains 18 internal variables and presents a very good accuracy in many well-known tests.

In terms of background contributions, enhanced strain elements have been established as a generalization of the so-called incompatible modes element [16] in Reference [17]. Other works have followed, such as the one in Reference [18] where the extension to finite strain plasticity was exposed and the 3D case was presented. Further work with the 3D case continued [19], to ensure the absence of volumetric locking. Further refinements of this classical formulation were carried out in Reference [20].

In classical enhanced strain elements hourglass instabilities occur in the finite strain regime when high levels of compression or tension are involved, a fact identified in Reference [21]. With the purpose of removing this defect, in References [11,19] a modified quadrature was proposed and in Reference [20] a stabilizing term and modified quadrature were adopted. More recently, in Reference [22], a penalty stabilizing term related to the original first Piola-Kirchhoff stress tensor was introduced by César de Sá et al.

Other stabilization approaches were used in References [23,24] in the context of the enhanced strain technique. Some noticeable enhanced strain contributions are the ones of Reference [8] where elements were tested with up to 30 internal variables and of [11] where 18 internal variables were adopted.

The enhanced strain technique is far from being the unique methodology to improve the accuracy of low-order elements. The reduced/average integration technique with various stabilization approaches $[9,14,25-30]$ is a cost-effective technique to improve elements' performance. Classical mixed formulations (assumed strains and/or stresses with internal variables) frequently result in high-accuracy solid elements (see References $[1,7,12,13,31-35]$ ) but few non-linear examples have been presented using these techniques. Moreover, the notion of 'selective scaling' is introduced in some exceptionally good performing elements [1] which somehow lacks generality. The so-called assumed strain formulation (some strain components are re-interpolated) which was introduced for plate and shell analysis by Bathe and co-workers (see the comprehensive expositions in References [36,37]) was also introduced into a hexahedral element in Reference [38], but in a shell analysis context.

To overcome the volumetric locking, some well-known solutions consist in adopting an independent pressure field and/or dilatation field. The family of $u-p$ (displacement-pressure), $\bar{B}$, mean-dilatation, selective-reduced integration, and related strategies is well-known and has several variations (see References [37,39-47]). These strategies are well adapted to nonlinear analysis and offer good results if bending is not involved. As a consequence, mixed $u-p$ strategies coupled with enhanced strain techniques have been proposed to overcome the deficient results in bending of classical $u-p$ elements (see References $[22,48,49]$ ) which are very cost-effective because fewer enhanced strain variables can be used. The adoption of 
both assumed stress and enhanced strains was also proposed in Reference [50] with reasonable results. The use of reduced/average integration with enhanced strains was proposed in References $[10,15,24]$ with success regarding the efficiency aspects.

A combination developed toward the analysis of strain softening problems was presented recently [51,52] and consists in the coupling of enhanced strain technique with an implicit gradient model.

Rather than inspecting a complex combination, the purpose of this work is to apply a classical enhanced strain technique, without any other tool to increase the performance of the element or reduce its cost. Additionally, the integration strategy is a standard Gauss quadrature with 8 points. Despite this simplicity, the results are very good in most situations and non-linear problems are dealt with success. The formulation contains a penalty stabilizing term which was established variationally (see Reference [22]). The element consists of an extension of the one proposed in References [51,52] where 9 internal variables instead of 18 were adopted and is identified as Hexahedral for near-Incompressibility and Shear (HIS). Some properties of the proposed element are:

- No special treatment or directional enhancement is adopted in the analysis of beams plates and shells. The same element can be used in both bulk forming, solid analysis, sheet metal forming and classical beam and plate linear analysis. Some solid-shell and 3D-shell (see Reference [38]) clearly identify the thickness direction and are unable to perform bulk solid analysis. On the other hand, some shell elements impose a plane stress condition to the material representation.

- There is no restriction concerning the constitutive law adopted (for instance $\bar{B}$ and $u-p$ elements contain a filter of the constitutive pressure, see Reference [22]).

- The formulation for general non-linear problems is fully detailed, exact linearization is performed and very good Newton-Raphson convergence properties are obtained, as shown in the examples.

- A hourglass control term is incorporated variationally (see References [51,22])

\section{FINITE ELEMENT FORMULATION}

The proposed finite element is based on the classical enhanced strain technique, with a particular selection of the enhanced strain shape functions and a derivation of the enhanced deformation gradient which ensures the Patch test satisfaction.

The static equilibrium condition of a given body $\mathfrak{B}$ follows for a conservative system. The extension to plasticity is carried out through the introduction of a scalar internal variable, which is the plastic multiplier, but the internal forces and consistent tangent stiffness matrix retain the form presented for the conservative system (see References [51,52] for further details).

An advantage of this approach is that it is guaranteed that the discretized system is conservative if the material behaviour of the body is elastic and the external loads are conservative.

Let $\Omega_{0}$ denote the volume integration domain corresponding to the non-deformed configuration of the body $\mathfrak{B}, \mathbf{x}$ the spatial position of an arbitrary point belonging to $\mathfrak{B}$ and $\mathbf{A}$ a certain second-order tensor. Additionally, $\mathbf{X}$ denotes the material position of an arbitrary point belonging to $\mathfrak{B}$. 
A total potential $\Pi$ based on two fields, $\mathbf{x}$ and $\mathbf{A}$, can be written as the sum of the potential elastic energy, $\Pi_{\text {int }}$ (internal energy) and the conservative external force potential, $\Pi_{\text {ext }}$ (external energy):

$$
\Pi(\mathbf{x}, \mathbf{A})=\underbrace{\int_{\Omega_{0}}[W\left(\nabla_{0} \mathbf{x}+\mathbf{A}\right)+\overbrace{\frac{r}{2} \mathbf{A}: \mathbf{A}}^{\Pi_{\mathrm{p}}}] \mathrm{d} v_{0}}_{\Pi_{\text {int }}}+\Pi_{\mathrm{ext}}(\mathbf{x})
$$

The external force potential $\Pi_{\text {ext }}$ is a function of the spatial position vector exclusively. The notation $W$ in (1) represents the strain energy function [47]. The tensor $\nabla_{0} \mathbf{x}=\partial \mathbf{x} / \partial \mathbf{X}$ is the continuum deformation gradient and $\mathrm{d} v_{0}=\mathrm{d} X_{1} \mathrm{~d} X_{2} \mathrm{~d} X_{3}$ where $X_{k}$ represent the scalar components of $\mathbf{X}$.

An important term in definition (1) is identified as $\Pi_{p}$ which, for $r \mapsto \infty$ imposes the nullity of $\mathbf{A}$ and hence the correspondence between definition (1) and the standard continuum potential (see References $[45,47,53]$ ). The determination of the parameter $r$ is only adopted in severe compression problems and it the minimum value with which hourglass does not occur. This determination is a drawback of most stabilization techniques for enhanced strain elements (see References [20,22]), of some stabilization techniques for reduced integration elements (see References $[25,26,28,29]$ ) and also of most stabilization techniques for the mixed $u-p$ formulations (References $[43,46]$ present some stabilization of $u-p$ low-order elements). In Reference [22] a connection between the penalty parameter $r$ and the first Piola-Kirchhoff stress tensor that acts as Lagrange multiplier in the original enhanced strain formulation [49] was established.

Form (1) of the total potential $\Pi$ is a particular case of a four-field potential presented recently [22].

If the body $\mathfrak{B}$ is discretized using NEL hexahedral elements, then the total potential $\Pi$ in (1) is approximated, due to discretization, as a sum of each element's contribution: $\Pi \cong \sum_{e=1}^{\mathrm{NEL}} \Pi^{e}$ where $\Pi^{e}=\Pi_{\mathrm{int}}^{e}+\Pi_{\mathrm{ext}}^{e}$. The internal energy corresponding to a certain element, $e$, with a Reference integration domain $V_{0}$ is written as

$$
\Pi_{\text {int }}^{e}=\int_{V_{0}}\left[W\left(\nabla_{0} \mathbf{x}+\mathbf{A}\right)+\frac{r}{2} \mathbf{A}: \mathbf{A}\right] \mathrm{d} V_{0}
$$

According to the procedure for the enrichment of the discretized deformation gradient (here denoted as $\mathbf{F}$ ), the following notation is used [22]:

$$
\mathbf{F}=\nabla_{0} \mathbf{x}+\mathbf{A}
$$

where $\nabla_{0} \mathbf{x}$ represents the material gradient of the spatial position vector and $\mathbf{A}$ is the already introduced term which is a function of the element's internal variables. In terms of scalar components, Equation (3) can be written as

$$
F_{i j}=\frac{\partial x_{i}}{\partial X_{j}}+A_{i j}
$$

where $i=1,2,3$ and $j=1,2,3$. 
If the nodal shape functions of the 8 node hexahedral are introduced

$$
N_{k}=0.125\left(1+\xi_{1 k} \xi_{1}\right)\left(1+\xi_{2 k} \xi_{2}\right)\left(1+\xi_{3 k} \xi_{3}\right)
$$

where $\xi_{l}$ represents the $l$-th local co-ordinate and $\xi_{k l}$ represents the value of the $l$-th local co-ordinate at the $k$-th node, we now have

$$
F_{i j}=\frac{\partial N_{k}}{\partial X_{j}} x_{k i}+A_{i j}
$$

In decomposition (6) $x_{k i}$ represent the spatial global co-ordinates of each node $k$ (similarly, $X_{k i}$ represent the material global co-ordinates of each node $k$ ).

If use is made of the Jacobian matrix which relates the material global co-ordinates with the local co-ordinates, whose scalar components are

$$
J_{i j}=\frac{\partial X_{i}}{\partial \xi_{j}}=\frac{\partial N_{k}}{\partial \xi_{j}} X_{k i}
$$

Equation (6) is re-written as

$$
F_{i j}=\frac{\partial N_{k}}{\partial \xi_{r}} J_{r j}^{-1} x_{k i}+A_{i j}
$$

In Reference [19], an approximate derivative of the hourglass part of the shape functions $N_{k}$ was carried out, instead of the conventional Equation (8). The argument is that with the particular approximation, the element remains '...locking free for distorted configurations (with non-constant Jacobian) in the incompressible limit' [19]. However, the approximation in Reference [19] causes the element to fail the Patch test (see Reference [15] for an alternative that guarantees the satisfaction of the Patch test).

To ensure the satisfaction of the Patch test, material averages are used. These conclusions have been numerically verified using the Patch test.

In this work, the following approximation is adopted:

$$
\frac{\partial N_{k}}{\partial X_{j}} \cong \overline{\left(\frac{\partial N_{k}}{\partial X_{j}}\right)}+\gamma_{k s} \frac{\bar{j}}{j} \frac{\partial H_{s}}{\partial \xi_{p}} \bar{J}_{p j}^{-1} \quad \text { with } \quad s=1, \ldots, 4
$$

where

$$
\begin{aligned}
\overline{\left(\frac{\partial N_{k}}{\partial X_{j}}\right)} & =\frac{1}{V_{0}} \int_{V_{0}} \frac{\partial N_{k}}{\partial X_{j}} \mathrm{~d} V_{0} \\
j & =\operatorname{det}[\mathbf{J}] \\
\bar{j} & =\frac{1}{V_{0}} \int_{V_{0}} j \mathrm{~d} V_{0} \\
\bar{J}_{p j}^{-1} & =\frac{1}{V_{0}} \int_{V_{0}} J_{p j}^{-1} \mathrm{~d} V_{0} \\
H_{1} & =\xi_{1} \xi_{2}, \quad H_{2}=\xi_{1} \xi_{3} \\
H_{3} & =\xi_{2} \xi_{3}, \quad H_{4}=\xi_{1} \xi_{2} \xi_{3}
\end{aligned}
$$


and $\gamma_{k s}$ with $s=1, \ldots, 3$ are the well-known stabilization vectors; see Reference [15, Equation (11)] or Reference [19, Equation (2.13a)] for a complete description.

A key development concerns the scalar components $A_{i j}$. If, in local co-ordinates, the term A is known through its mixed-variant scalar components $\tilde{A}_{q t}$, then it follows that

$$
A_{r s}=\frac{\partial x_{r}}{\partial X_{p}} J_{p q} \tilde{A}_{q t} J_{t s}^{-1}
$$

However, to ensure that the following condition (see References [17-19,22]) is satisfied

$$
\int_{V_{0}} \mathbf{A} \mathrm{d} V_{0}=\mathbf{0}
$$

for a given selection of the scalar components $\tilde{A}_{p q}$, the terms $J_{p q}, J_{t s}^{-1}$ and $\partial x_{r} / \partial X_{p}$ must be independent of the local co-ordinates $\xi_{l}$.

Therefore, material averages of these quantities are used:

$$
A_{r s}=\frac{\bar{j}}{j} \bar{F}_{r p} \bar{J}_{p q} \tilde{A}_{q t} \bar{J}_{t s}^{-1}
$$

where

$$
\bar{F}_{r p}=\frac{1}{V_{0}} \int_{V_{0}} \frac{\partial x_{r}}{\partial X_{p}} \mathrm{~d} V_{0}
$$

and

$$
\bar{J}_{p q}=\frac{1}{V_{0}} \int_{V_{0}} J_{p q} \mathrm{~d} V_{0}
$$

Having established relation (17) it is important to ensure that the condition $\int_{-1}^{1} \int_{-1}^{1} \int_{-1}^{1} \tilde{A}_{q t}$ $\mathrm{d} \xi_{1} \mathrm{~d} \xi_{2} \mathrm{~d} \xi_{3}=0$ is satisfied, so that the Patch test is satisfied (see References [17-19]).

The particular form for $\tilde{A}_{q t}$ can be presented as

$$
\tilde{A}_{q t}=\tilde{E}_{q t z} \alpha_{z}
$$

where $\alpha_{z}$ are the enhanced strain variables, which represent variables internal to the element.

The term $\tilde{E}_{q t z}$ in Equation (20) can be written according to Table I, where

$$
M_{k}=\frac{\partial}{\partial \xi_{k}}\left[\left(1-\xi_{1}^{2}\right)\left(1-\xi_{2}^{2}\right)\left(1-\xi_{3}^{2}\right)\right]
$$

represent the local co-ordinate derivatives of the $3 \mathrm{D}$ bubble function. Motivations to use this function are discussed in Reference [22].

A 3D element based on this function was published recently [51]. The first 9 shape functions in Table I are a simple extension of the ones proposed for the $2 \mathrm{D}$ case in a recent publication [22] and applied for the first time in a 3D case in Reference [51]. These are related to the bending modes of the hexahedral. The remaining 9 are related to the torsion modes and only make sense in the present $3 \mathrm{D}$ case. 
Table I. Table of enhanced strain shape functions in terms of scalar components of $\tilde{E}_{q t z}$.

\begin{tabular}{lcc}
\hline$\tilde{E}_{q t 1}=M_{1} \delta_{q t} \delta_{t 1}$ & $\tilde{E}_{q t 2}=M_{2} \delta_{q t} \delta_{t 2}$ & $\tilde{E}_{q t 3}=M_{3} \delta_{q t} \delta_{t 3}$ \\
$\tilde{E}_{q t 4}=M_{2} \delta_{q 1} \delta_{t 2}$ & $\tilde{E}_{q t 5}=M_{1} \delta_{q 2} \delta_{t 1}$ & $\tilde{E}_{q t 6}=M_{3} \delta_{q 1} \delta_{t 3}$ \\
$\tilde{E}_{q t 7}=M_{1} \delta_{q 3} \delta_{t 1}$ & $\tilde{E}_{q t 8}=M_{3} \delta_{q 2} \delta_{t 3}$ & $\tilde{E}_{q t 9}=M_{2} \delta_{q 3} \delta_{t 2}$ \\
$\tilde{E}_{q t 10}=M_{2} M_{3} \delta_{q t} \delta_{t 1}$ & $\tilde{E}_{q t 11}=M_{1} M_{3} \delta_{q t} \delta_{t 2}$ & $\tilde{E}_{q t 12}=M_{1} M_{2} \delta_{q t} \delta_{t 3}$ \\
$\tilde{E}_{q t 13}=M_{3} M_{2} \delta_{q 1} \delta_{t 2}$ & $\tilde{E}_{q t 14}=M_{3} M_{1} \delta_{q 2} \delta_{t 1}$ & $\tilde{E}_{q t 15}=M_{2} M_{3} \delta_{q 1} \delta_{t 3}$ \\
$\tilde{E}_{q t 16}=M_{2} M_{1} \delta_{q 3} \delta_{t 1}$ & $\tilde{E}_{q t 17}=M_{1} M_{3} \delta_{q 2} \delta_{t 3}$ & $\tilde{E}_{q t 18}=M_{1} M_{2} \delta_{q 3} \delta_{t 2}$ \\
\hline
\end{tabular}

With the purpose of calculating the element's internal forces and consistent tangent stiffness matrix, the following notations are introduced

$$
\begin{aligned}
\check{A}_{i j} & =\bar{J}_{i r} \tilde{A}_{r s} \bar{J}_{s j}^{-1} \\
F_{i j} & =\bar{F}_{i u} \check{A}_{u j} \frac{\bar{j}}{j}+\frac{\partial x_{i}}{\partial X_{j}} \\
\check{E}_{k i j} & =\bar{J}_{i r} \tilde{E}_{k r s} \bar{J}_{s j}^{-1} \\
E_{k i j} & =\bar{F}_{i u} \check{E}_{k u j} \frac{\bar{j}}{j}
\end{aligned}
$$

The spatial velocity gradient, which is identified as $\mathbf{L}$, is represented by its scalar components according to

$$
L_{i j}=\dot{F}_{i r} F_{r j}^{-1} \Leftrightarrow L_{i j}=\underbrace{\left[\frac{\partial N_{k}}{\partial X_{r}} F_{r j}^{-1}+\frac{\bar{j}}{\bar{j}\left(\frac{\partial N_{k}}{\partial X_{u}}\right)} \check{F}_{u r} F_{r j}^{-1}\right]}_{\hat{N}_{k j}} \dot{x}_{k i}+\underbrace{\frac{\bar{j}}{j} \bar{F}_{i u} \check{E}_{k u r} F_{r j}^{-1}}_{\hat{G}_{k i j}} \dot{\alpha}_{k}
$$

where the notation $\dot{x}_{k i}$ represents the time derivatives of the nodal spatial position and the terms $\alpha_{k}$ identify the time derivative of the enhanced strain variable with index $k$.

Equation (26) provides a definition of the terms $\hat{N}_{k j}$ and $\hat{G}_{k i j}$, which relate the spatial velocity gradient with the nodal spatial co-ordinates and the enhanced strain variables, respectively.

If the equilibrium condition at the element level is established using the stationarity of $\Pi^{e}$, $\delta \Pi^{e}=0$, and then

$$
\delta \Pi^{e}=0 \Leftrightarrow \int_{V_{0}} \tau_{i j} \hat{N}_{k j} \delta x_{k i} \mathrm{~d} V_{0}+\int_{V_{0}} \tau_{i j} \hat{G}_{k i j} \delta \alpha_{k} \mathrm{~d} V_{0}+\int_{V_{0}} r A_{i j} \delta A_{i j} \mathrm{~d} V_{0}+\delta_{x} \Pi_{\text {ext }}^{e}=0
$$

with $\tau_{i j}$ being the Kirchhoff stress tensor scalar components, related with the Cauchy stress tensor components, $\sigma_{i j}$ by $\sigma_{i j}=\tau_{i j} / \operatorname{det}[\mathbf{F}]$. The relation between $W(\mathbf{F})$ and $\tau$ is $\tau=\partial_{\mathbf{F}} W \mathbf{F}^{\mathrm{T}}$.

From (27) the element's internal forces and external forces are calculated (for a standard calculation of external forces see Reference [37]). A note regarding Equation (27) is that the contribution $\delta \Pi_{\text {ext }}^{e}$ is exclusively due to the change in the spatial nodal co-ordinates. 
The calculation of the consistent tangent stiffness, which is a necessity of the NewtonRaphson method, is based on the second variation of $\Pi^{e}: \mathrm{d} \delta \Pi^{e}$. Here use is made of the consistent modulus, $\mathbf{C}_{T}$, relative to the Truesdell rate of the Kirchhoff stress $\dot{\tau}^{\mathrm{Tr}}$, is

$$
\dot{\tau}^{\operatorname{Tr}}=\mathbf{C}_{T}: \mathbf{D}
$$

where $\mathbf{D}=\frac{1}{2}\left(\mathbf{L}+\mathbf{L}^{\mathrm{T}}\right)$ represents the rate of deformation tensor (see also References $\left.[47,53]\right)$.

The Truesdell rate of the Kirchhoff stress is related to the time derivative of the Kirchhoff stress tensor, $\dot{\tau}$ (see also Reference [22]):

$$
\dot{\tau}^{\mathrm{Tr}}=\dot{\tau}-\mathbf{L} \tau-\tau \mathbf{L}^{\mathrm{T}}
$$

Using this notion, the second variation of $\Pi_{\mathrm{int}}^{e}$ is calculated

$$
\begin{aligned}
\mathrm{d} \delta \Pi_{\text {int }}^{e}= & \int_{V_{0}} C_{T i j r s} \hat{N}_{k j} \hat{N}_{l s} \mathrm{~d} x_{l r} \delta x_{k i} \mathrm{~d} V_{0}+\int_{V_{0}} C_{T i j r s} \hat{G}_{m r s} \hat{G}_{n i j} \mathrm{~d} \alpha_{m} \delta \alpha_{n} \mathrm{~d} V_{0} \\
& +\int_{V_{0}}\left(r \mathrm{~d} A_{i j} \delta A_{i j}+r A_{i j} \mathrm{~d} \delta A_{i j}\right) V_{0} \\
& +\int_{V_{0}}\left(\hat{N}_{l j} \mathrm{~d} x_{l i}+\hat{G}_{m i j} \mathrm{~d} \alpha_{m}\right)\left(\hat{N}_{k s} \delta x_{k i}+\hat{G}_{n i s} \delta \alpha_{n}\right) \tau_{j s} \mathrm{~d} V_{0}
\end{aligned}
$$

where $i, j, r, s$ indicate degrees of freedom, $k, l$ indicate nodes and $m, n$ indicate enhanced strain variables.

The implementation of element HIS is straightforward, and follows directly from Equation (27) for the forces and from Equation (30) for the consistent tangent stiffness.

\section{NUMERICAL EXAMPLES}

The following examples illustrate the application of the derivations carried out in Section 2 in both linear and non-linear situations. The purpose is to validate the present element formulation in a wide range of situations.

Firstly, some variants of the Patch test are satisfied. After that, several linear elastic tests are performed. Finally, various classical problems with geometric non-linearities and material non-linearities are presented.

The elasto-plastic model adopted in several problems has been described in References $[51,52]$ and is a variant of the one adopted in other sources $[49,54,55]$. For the purely elastic analysis, the Hencky hyperelastic model is adopted, with exception of the silicone rubber tension test where the Yeoh hyperelastic model is employed (see Reference [47]).

A standard Newton-Raphson method with bisection line-search is used in most non-linear computations, and, in problems involving instabilities, the arc-length method discussed in Reference [56] is employed, with a linearized treatment if no real roots are obtained.

All the computations were carried out in code SIMPLAS, a Fortran 90 code developed by the first author of this work. 


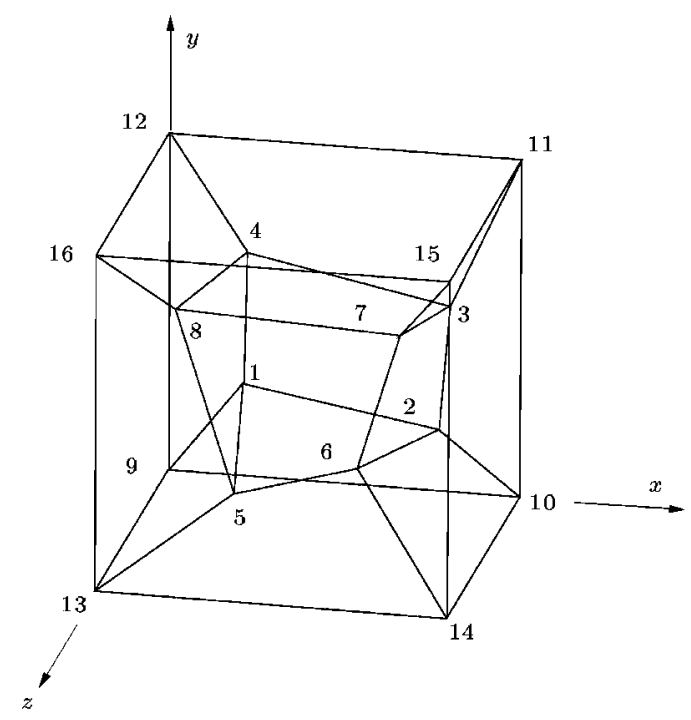

Figure 1. The Patch test for solids, according to Reference [57].

Note that in all the examples except the last one, there was no need of using the stabilization term.

Therefore $r=0$ in problems from 3.1 to 3.3 .9 and $r=1.5 E / 2(1+v)$ in problem 3.3.10.

\subsection{Patch test}

The Patch test for solids suggested in Reference [57] is here explored. The purpose of this test is to verify the correctness of the computer implementation of the element. Displacements are applied at the exterior nodes to induce zero stresses in rigid body motion (Case A), constant stresses (Case B) and linear varying displacements (Case C). Figure 1 shows a unit cube containing 7 hexahedral distorted elements. The elasticity modulus considered $E$ is $1 \times 10^{6}$ consistent units and the Poisson coefficient $v$ is 0.25 .

For Figure 1, the Case $\mathrm{A}$ is materialized by a rigid body rotation $\theta_{z}=45^{\circ}$ along the axis defined by nodes $9-13$ through the imposition of a displacement $v=1 / \sqrt{2}$ at nodes $10-14$ and also by a rigid body translation through the movement of face 10-11-14-15, through a displacement value $u=1$.

These two rigid body motions of Case A involve large displacements, and are sufficient for checking if stress values near zero are obtained.

Case $\mathrm{B}$ consists in imposing a pure shear through the application of shear forces at the nodes of face 10-11-14-15, with $f_{y}=1 \times 10^{2}$ and pure tension through the application of normal forces at face 10-11-14-15, with $f_{x}=1 \times 10^{2}$.

Finally, case $\mathrm{C}$ is tested imposing the following displacement field at the exterior nodes only $(9, \ldots, 16)$ :

$$
\begin{array}{r}
u=5 \times 10^{-4}(2 x+y+z) \\
v=5 \times 10^{-4}(x+2 y+z) \\
w=5 \times 10^{-4}(x+y+2 z)
\end{array}
$$


Table II. Patch test, Case A: Large amplitude rigid body motion (rotation and translation).

\begin{tabular}{llll}
\hline Stress component & Max. value & Min. value & Theory \\
\hline Rotation, $\theta_{z}=45^{\circ}$ & & & \\
$\tau_{11}$ & $9.96 \times 10^{-10}$ & $-1.13 \times 10^{-9}$ & 0 \\
$\tau_{22}$ & $1.09 \times 10^{-9}$ & $-8.17 \times 10^{-10}$ & 0 \\
$\tau_{33}$ & $5.82 \times 10^{-10}$ & $-7.22 \times 10^{-10}$ & 0 \\
$\tau_{12}$ & $4.17 \times 10^{-10}$ & $-5.25 \times 10^{-10}$ & 0 \\
$\tau_{13}$ & $1.23 \times 10^{-10}$ & $-1.90 \times 10^{-10}$ & 0 \\
$\tau_{23}$ & $2.01 \times 10^{-10}$ & $-2.25 \times 10^{-10}$ & 0 \\
$T_{r a n s l a t i o n,} u=1$ & & & \\
$\tau_{11}$ & $1.07 \times 10^{-9}$ & $-8.46 \times 10^{-10}$ & 0 \\
$\tau_{22}$ & $9.45 \times 10^{-10}$ & $-7.09 \times 10^{-10}$ & 0 \\
$\tau_{33}$ & $1.00 \times 10^{-9}$ & $-1.14 \times 10^{-9}$ & 0 \\
$\tau_{12}$ & $2.26 \times 10^{-10}$ & $-2.21 \times 10^{-10}$ & 0 \\
$\tau_{13}$ & $4.37 \times 10^{-10}$ & $-3.76 \times 10^{-10}$ & 0 \\
$\tau_{23}$ & $1.91 \times 10^{-10}$ & $-2.74 \times 10^{-10}$ & 0 \\
\hline
\end{tabular}

Table III. Patch test, Case B: Shear and tension.

\begin{tabular}{lccc}
\hline Stress component & Max. value & Min. value & Theory \\
\hline Shear, $\tau_{12}=400$ & 0 & 0 & \\
$\tau_{11}$ & 0 & 0 & 0 \\
$\tau_{22}$ & 0 & 0 & 0 \\
$\tau_{33}$ & 400 & 400 & 400 \\
$\tau_{12}$ & 0 & 0 & 0 \\
$\tau_{13}$ & 0 & 0 & 0 \\
$\tau_{23}$ & & & \\
Tension, $\tau_{11}=400$ & 400 & 400 & 400 \\
$\tau_{11}$ & 0 & 0 & 0 \\
$\tau_{22}$ & 0 & 0 & 0 \\
$\tau_{33}$ & 0 & 0 & 0 \\
$\tau_{12}$ & 0 & 0 & 0 \\
$\tau_{13}$ & 0 & 0 & 0 \\
$\tau_{23}$ & 0 &
\end{tabular}

For Case A, the residual stresses exposed in Table II are extremely small relative to the elasticity modulus and can be considered zero. For Case B, the values in Table III are exact both for shear and tension situations.

For Case C, whose results are presented in Table IV, the analytical values are reproduced exactly, a condition established in Reference [57] and known to ensure convergence of results as mesh is refined (see also the explanatory considerations in Chapter 9 of Reference [58]). 
Table IV. Patch test, Case C.

\begin{tabular}{|c|c|c|c|c|c|c|}
\hline \multirow[b]{2}{*}{ Node } & \multicolumn{3}{|c|}{ Analytical } & \multicolumn{3}{|c|}{ Numerical } \\
\hline & $u$ & $v$ & $w$ & $u$ & $v$ & $w$ \\
\hline 1 & $5.16 \times 10^{-4}$ & $5.63 \times 10^{-4}$ & $4.87 \times 10^{-4}$ & $5.16 \times 10^{-4}$ & $5.63 \times 10^{-4}$ & $4.87 \times 10^{-4}$ \\
\hline 2 & $1.11 \times 10^{-3}$ & $8.45 \times 10^{-4}$ & $8.45 \times 10^{-4}$ & $1.11 \times 10^{-3}$ & $8.45 \times 10^{-4}$ & $8.45 \times 10^{-4}$ \\
\hline 3 & $1.31 \times 10^{-3}$ & $1.21 \times 10^{-3}$ & $1.01 \times 10^{-3}$ & $1.31 \times 10^{-3}$ & $1.21 \times 10^{-3}$ & $1.01 \times 10^{-3}$ \\
\hline 4 & $7.63 \times 10^{-4}$ & $1.00 \times 10^{-3}$ & $7.42 \times 10^{-4}$ & $7.63 \times 10^{-4}$ & $1.00 \times 10^{-3}$ & $7.42 \times 10^{-4}$ \\
\hline 5 & $7.35 \times 10^{-4}$ & $6.68 \times 10^{-4}$ & $8.96 \times 10^{-4}$ & $7.35 \times 10^{-4}$ & $6.68 \times 10^{-4}$ & $8.96 \times 10^{-4}$ \\
\hline 6 & $1.17 \times 10^{-3}$ & $9.85 \times 10^{-4}$ & $1.17 \times 10^{-3}$ & $1.17 \times 10^{-3}$ & $9.85 \times 10^{-4}$ & $1.17 \times 10^{-3}$ \\
\hline 7 & $1.46 \times 10^{-3}$ & $1.41 \times 10^{-3}$ & $1.38 \times 10^{-3}$ & $1.46 \times 10^{-3}$ & $1.41 \times 10^{-3}$ & $1.38 \times 10^{-3}$ \\
\hline 8 & $8.88 \times 10^{-4}$ & $1.18 \times 10^{-3}$ & $1.16 \times 10^{-3}$ & $8.88 \times 10^{-4}$ & $1.18 \times 10^{-3}$ & $1.16 \times 10^{-3}$ \\
\hline
\end{tabular}
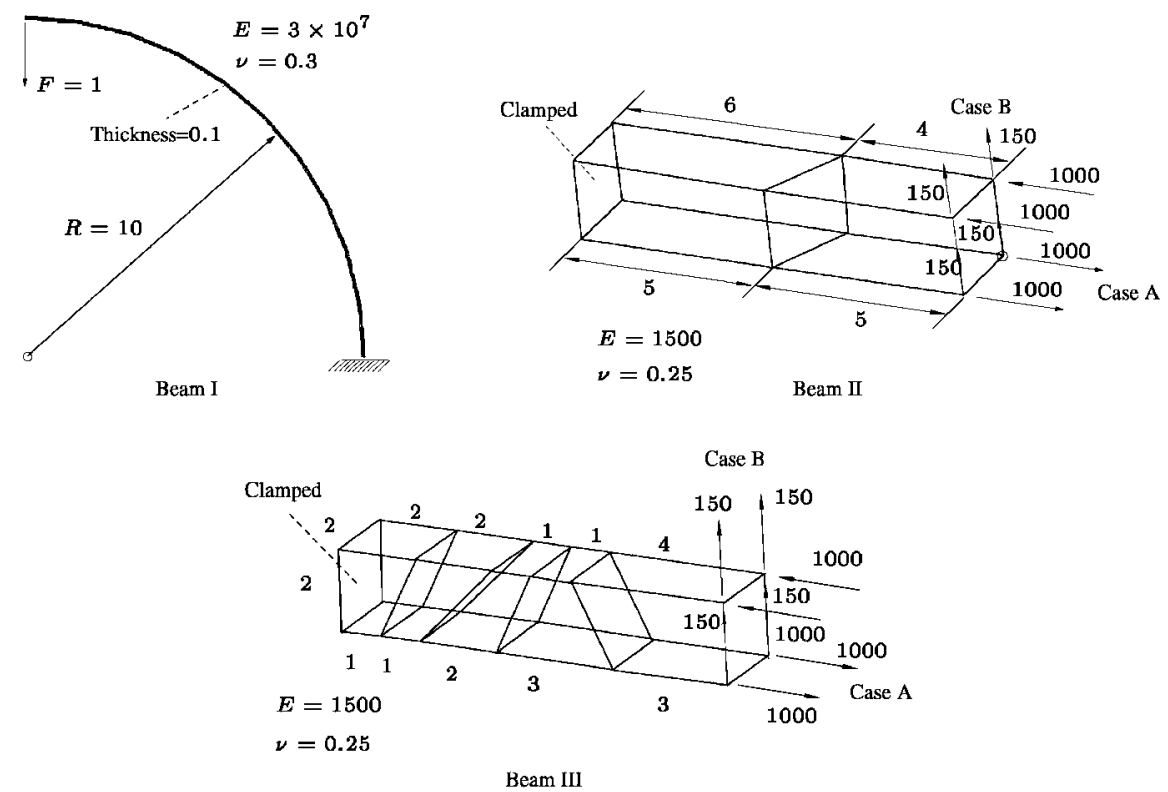

Figure 2. Linear elastic case: set of beams analysed. Beam I is a curved beam, beams II and III are distorted cantilever beams.

\subsection{Linear problems}

The usefulness of the following tests lie in the inspection of the accuracy of the proposed element in the linear case. The suitability of the new 3D element in situations that are typically analysed with beam, plate and shell elements is verified. Some well-known solutions, from numerical and analytical sources are used in the comparisons. A near-incompressible problem with mesh distortion, which is known to be a difficult problem for low-order 3D elements is also presented.

3.2.1. Beam problems. Figure 2 shows the geometry, boundary conditions and elastic material properties for the tested beams. 
Table V. Beam I: convergence of normalized results.

\begin{tabular}{lcc}
\hline Mesh & HIS & Reference [9] \\
\hline $5 \times 1 \times 1$ & 0.8418 & 0.787 \\
$10 \times 2 \times 2$ & 0.9955 & 0.998 \\
$20 \times 4 \times 4$ & 1.012 & 1.023 \\
\hline
\end{tabular}

Table VI. Beam II: Normalized results.

\begin{tabular}{lccc}
\hline Case & HIS & QM/E12 [10] & QSN/E9 [10] \\
\hline A & 0.924 & 0.901828 & 0.892957 \\
B & 0.882 & 0.851621 & 0.846237 \\
\hline
\end{tabular}

Table VII. Beam III: Normalized results.

\begin{tabular}{lcccc}
\hline Case & HIS & QM/E12 [10] & QSN/E9 [10] & Pian 1984 [31] \\
\hline A & 0.9583 & 0.9299 & 0.9953 & 0.9618 \\
B & 0.9535 & 0.9237 & 0.9903 & 0.9570 \\
\hline
\end{tabular}

The curved beam (beam I) was tested in Reference [47] and a target solution for the displacement under load of $v=3.14159$ is adopted. The normalized displacement under load is presented in Table $\mathrm{V}$ where the results taken from Reference [9] are the ones of the element NUHEXIN-4. Although the element from the above reference presents slightly more accuracy for the $10 \times 2 \times 2$ mesh, it tends to be too soft when finer meshes are used (in a lesser extent the same occurs with element HIS).

Beam II is presented in Reference [10] where the element QSN/E9 was introduced. The same reference analyses the element QM/E12 proposed in Reference [19] in the present problem. For case A in Figure 2 the solution for the transverse displacement is $v=100$, and for case $\mathrm{B}$ the solution is $v=102.6$. The point indicated with a small circle in Figure 2 is monitored. The results are presented in Table VI. It is clear that, for this test, the proposed HIS element is the most accurate.

Beam III is a well-known test (see References [10,31]) with the solution values being the same as those presented for beam II. Table VII shows the normalized results. In Reference [31] a $2 \mathrm{D}$ element was used.

Although in this case (in contrast with the case of beam II) QSN/E9 gives more accurate results than HIS, the results for HIS element are very close to the ones obtained using a 2D hybrid formulation (see Reference [31]).

3.2.2. Plate problems. Figures 3 and 4 show the geometries and boundary conditions for various problems of plate bending. Plate I is a clamped circular plate subject to a point load, plate II is a clamped square plate subject to point load, plate III is a simply supported square plate subject to point load and plate IV is a simply supported $30^{\circ}$ skew plate subject to uniform pressure. Two plates discretized with distorted meshes are shown in Figure 4. 

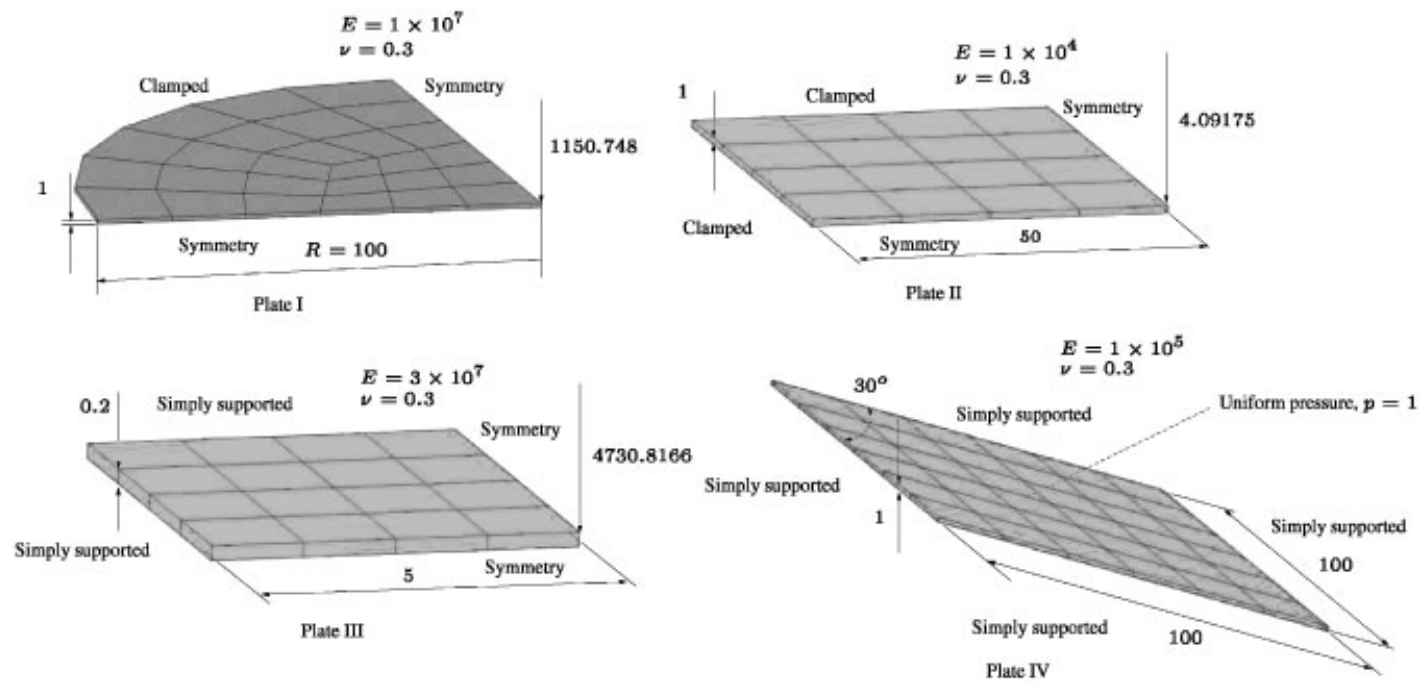

Figure 3. Linear elastic case: set of non-distorted plates analysed.

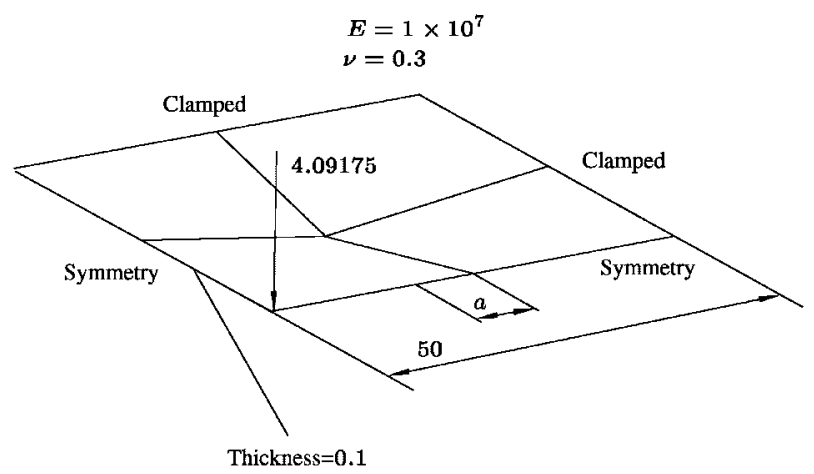

Plate with distorted mesh I

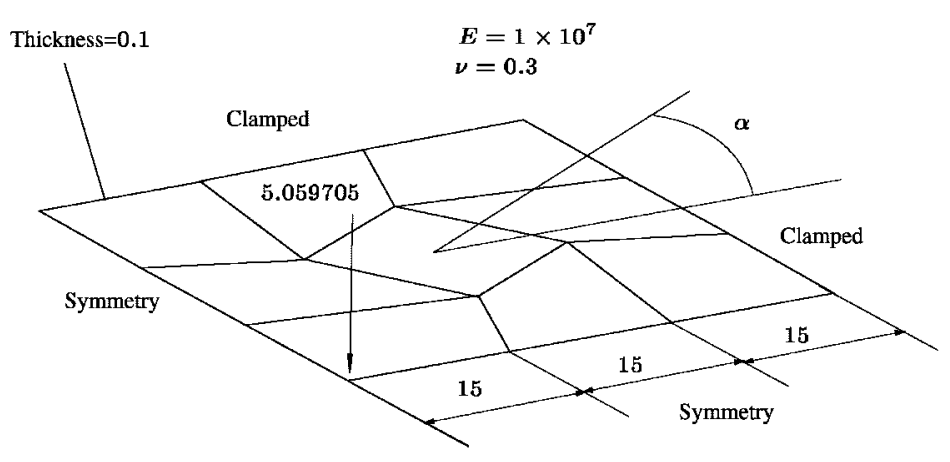

Plate with distorted mesh II

Figure 4. Linear elastic case: set of distorted square plates analysed. 


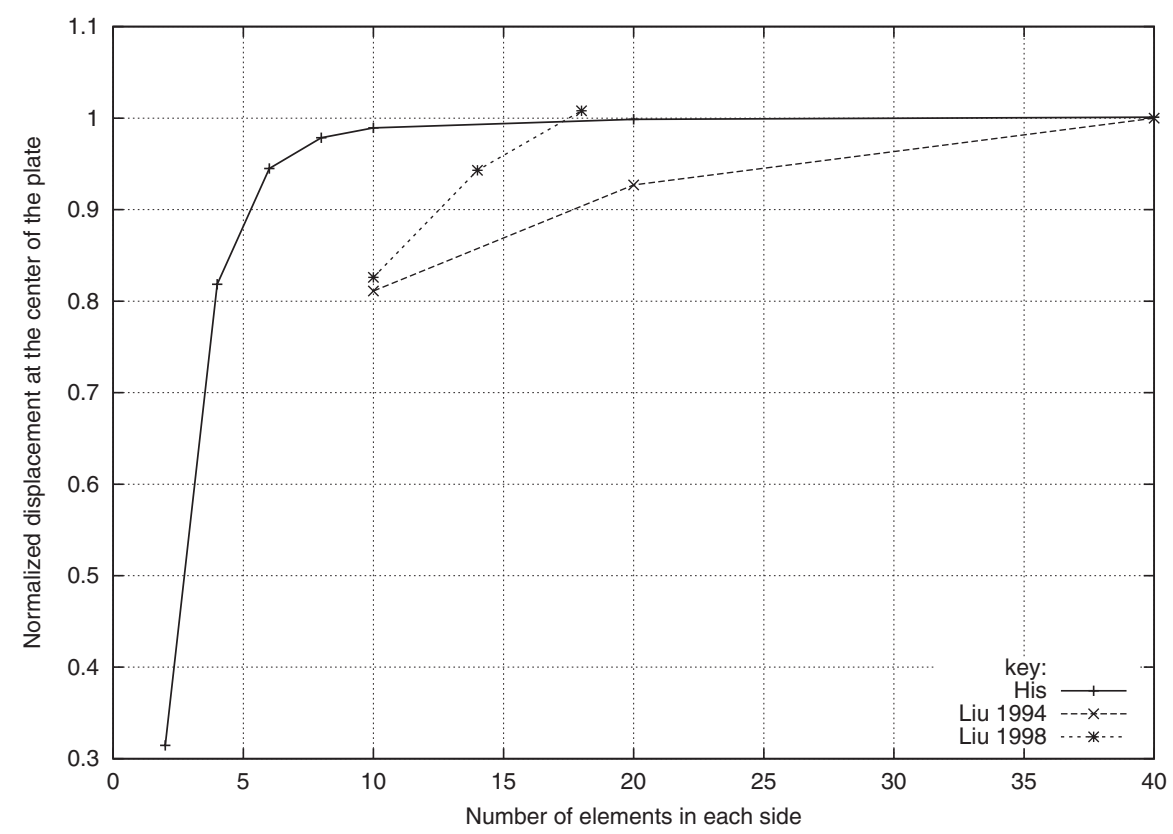

Figure 5. Plate I: convergence of normalized displacement as a function of the number of elements in each side, results from References $[9,14]$ are presented.

A normalization of results with Kirchhoff plate theory (see Reference [59]) is carried out in all the plates, except in plate IV, where a different target value is adopted (see Reference [8]).

The solution of the circular plate problem is specially important, since the clamped boundary is grossly represented by straight-edged elements. The best results obtained with reduced integration hexahedral elements (see References $[9,14]$ ) are presented for comparison in Figure 5. For Reference [9], the best results were obtained with their NUHEXIN-4 element, and these are presented here for comparison. Table VIII shows the results and it is being noticeable that with the present HIS formulation, there is no need for adopting several elements along the thickness, as is the case with References [9, 14].

It is clear from Figure 5 and Table VIII that element HIS presents very accurate and fast convergent results, clearly outperforming the elements from References [9, 14].

The results for the clamped square plate (plate II) obtained with the present element and the results from References [8,12] are all presented in Figure 6. The results from Reference [8] correspond to their 3D.EAS-30 element, which contains 30 internal variables. Although the clamped plate in Reference [12] presents distinct dimensions, the aspect ratio length/thickness $L / t=100$ is the same and therefore, as the Poisson coefficient $v$ is also 0.3 , the comparison is valid. It is noticeable that the mixed element employed in Reference [12] presents apparently fast converging results, but in fact it converges to a higher value than the theoretical solution.

From the observation of Figure 6 and Table IX, it is clear that the best overall results are obtained with the present element. 
Table VIII. Plate I: normalized displacement as a function of the number of elements in each side.

\begin{tabular}{lccc}
\hline Elements/side & HIS & Liu et al. $[9]$ & Liu et al. $[14]$ \\
\hline 2 & 0.315 & $*$ & $*$ \\
4 & 0.818 & $*$ & $*$ \\
6 & 0.945 & $*$ & $*$ \\
8 & 0.979 & $*$ & $*$ \\
10 & 0.989 & $0.811,2$ thick. elem. & $0.826,2$ thick. elem. \\
14 & $*$ & $*$ & $0.943,2$ thick. elem. \\
18 & $*$ & $*$ & $1.008,2$ thick. elem. \\
20 & 0.998 & $0.927,4$ thick. elem. & $*$ \\
40 & 1.001 & $1.000,4$ thick. elem. & $*$ \\
\hline
\end{tabular}

$*=$ non-available data.

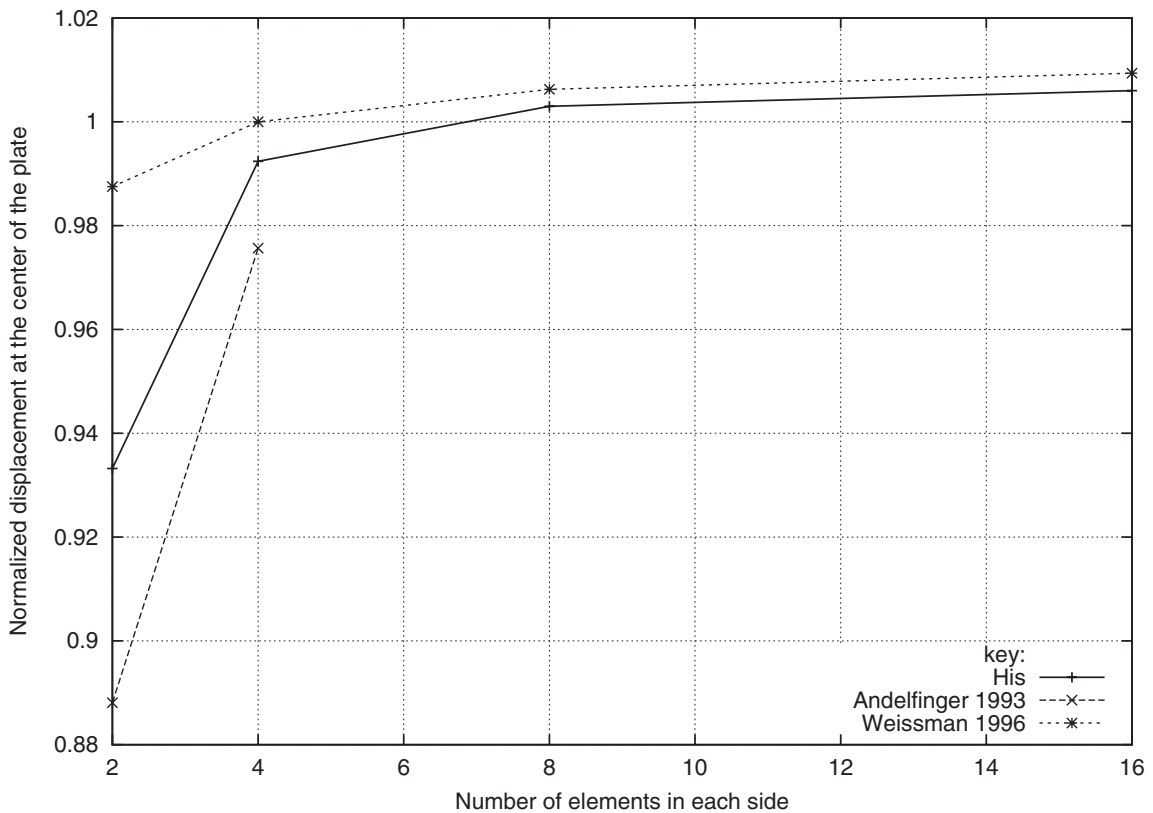

Figure 6. Plate II: convergence of normalized displacement as a function of the number of elements on each side.

Table IX. Plate II: convergence of normalized displacement.

\begin{tabular}{lcc}
\hline Elements/side & HIS & Andelfinger [8] \\
\hline 2 & 0.933 & 0.888 \\
4 & 0.992 & 0.976 \\
8 & 1.003 & $*$ \\
16 & 1.006 & $*$ \\
\hline
\end{tabular}




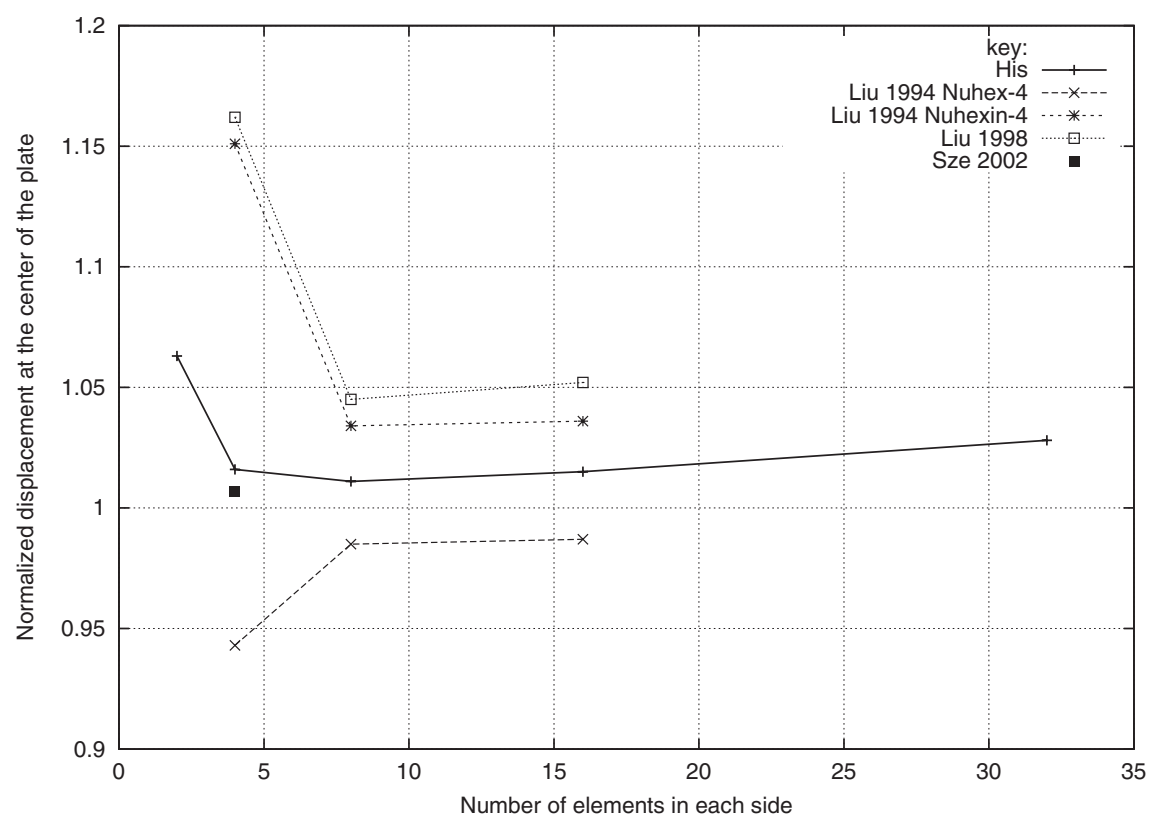

Figure 7. Plate III: convergence of normalized displacement as a function of the number of elements on each side.

Table X. Plate III: convergence of normalized displacement.

\begin{tabular}{|c|c|c|c|c|c|}
\hline Elements/side & HIS & $\begin{array}{l}\text { Liu et al., } \\
\text { NUHEX-4 [9] }\end{array}$ & $\begin{array}{l}\text { Liu et al., } \\
\text { NUHEXIN-4 [9] }\end{array}$ & Liu et al. [14] & Sze et al. [7] \\
\hline 2 & 1.063 & * & $*$ & $*$ & $*$ \\
\hline 4 & 1.016 & 0.943 & $\begin{array}{l}1.151, \\
2 \text { thick. } \\
\text { elem. }\end{array}$ & $\begin{array}{l}1.162 \\
2 \text { thick. } \\
\text { elem. }\end{array}$ & 1.007 \\
\hline 8 & 1.011 & 0.985 & $\begin{array}{l}1.034 \\
4 \text { thick. } \\
\text { elem. }\end{array}$ & $\begin{array}{l}1.045 \\
4 \text { thick. } \\
\text { elem. }\end{array}$ & * \\
\hline 16 & 1.015 & 0.987 & $\begin{array}{l}1.036 \\
4 \text { thick. } \\
\text { elem. }\end{array}$ & $\begin{array}{l}1.052 \\
4 \text { thick. } \\
\text { elem. }\end{array}$ & * \\
\hline 32 & 1.028 & $*$ & $*$ & $*$ & $*$ \\
\hline
\end{tabular}

The results for the simply supported square plate, denoted as plate III, are compared with the results from References [9, 14] and [7]. The plate in Reference [7] has different dimensions, but the same aspect ratio $L / t=50$, so this comparison is legitimate.

Figure 7 shows the comparison, and Table $\mathrm{X}$ shows the excellent performance of the HIS element, with a single value for the displacement as provided by Reference [7] being clearly insufficient to question the superiority of the present element in this example. 


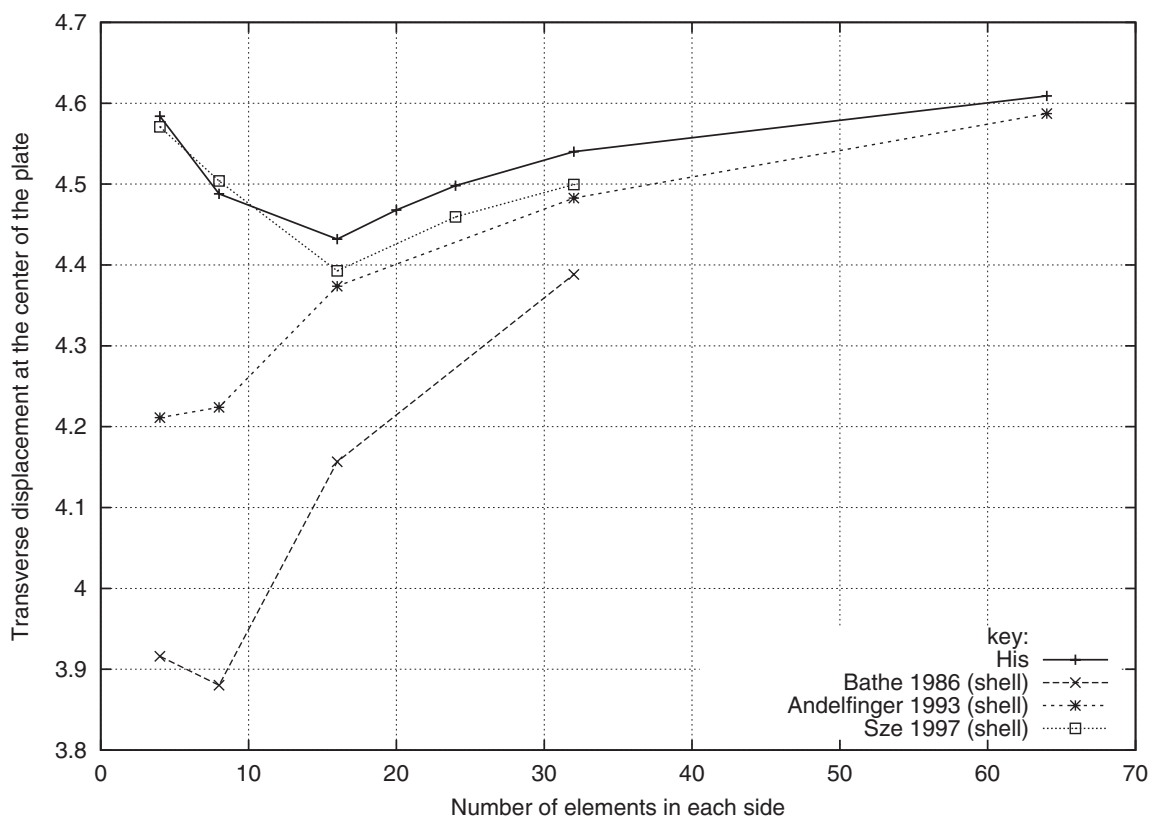

Figure 8. Plate IV: displacement at the centre of the skew plate as a function of the number of elements in each side, comparison with References $[8,33,36]$.

Table XI. Plate IV: displacement at the centre of the skew plate.

\begin{tabular}{lcccc}
\hline Elements/side & HIS & $\begin{array}{c}\text { Bathe and } \\
\text { Dvorkin [36] }\end{array}$ & $\begin{array}{c}\text { Andelfinger and } \\
\text { Ramm [8] }\end{array}$ & Sze et al. [33] \\
\hline 4 & 4.584 & 3.916 & 4.212 & 4.571 \\
8 & 4.488 & 3.880 & 4.224 & 4.504 \\
16 & 4.432 & 4.157 & 4.374 & 4.392 \\
20 & 4.468 & $*$ & $*$ & $*$ \\
24 & 4.498 & $*$ & $*$ & 4.459 \\
32 & 4.540 & 4.388 & 4.483 & $* .500$ \\
64 & 4.609 & $*$ & 4.587 & $*$ \\
\hline
\end{tabular}

For the skew plate, the target displacement at the centre of the plate with a value of 4.64 is to be expected, as stated in Reference [8]. Comparison with References [8,33,36] is carried out. The values of Reference [8] are the ones of shell element EAS7-ANS. Figure 8 shows the convergence of the results in a graphical form, and Table XI shows the same results but in tabular form. From the observation of the figure and the table, it is clear that HIS element presents the most accurate results, much better than well-known shell formulations, such as Bathe's MITC4 element [36].

Finally, to study the plate mesh distortion effect, the results for the plates represented in Figure 4 are shown in Figure 9, along with the results from Reference [60]. 

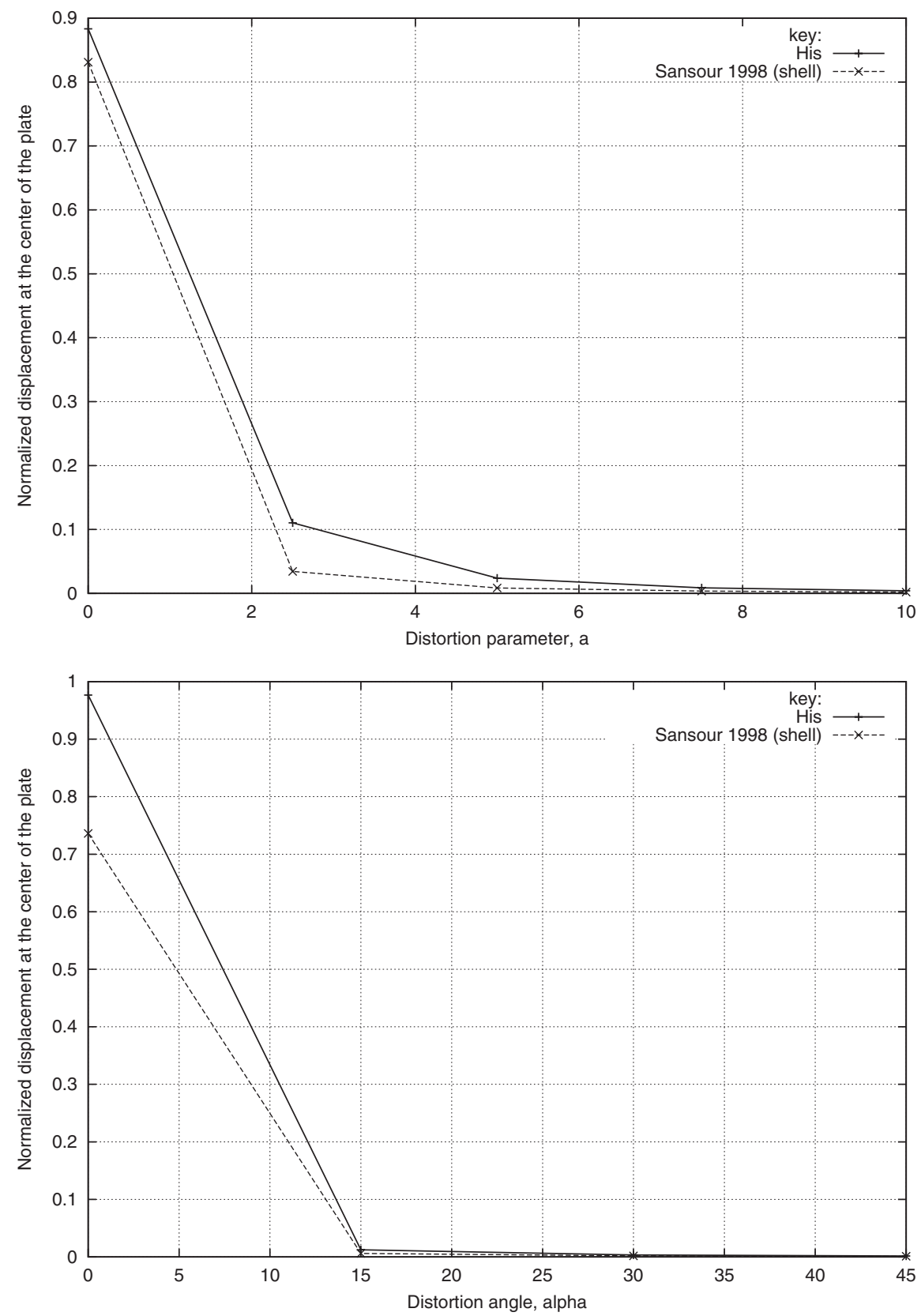

Figure 9. The effect of mesh distortion in plate bending (see Figure 4). In the first plate, a node is displaced toward the point load, and in the second plate, a rotation of a central element is imposed. The results of Reference [60] are presented for comparison. 
Despite the large errors involved, Figure 9 clearly shows the superiority of HIS element in this example.

3.2.3. Shell problems. The analysis of the 5 shell problems illustrated in Figure 10 follows.

Shell I consists on a pinched cylinder and has been analysed, among others, in References $[4,10,12,14,50,61]$, whose results are here reproduced. A reference value of $1.82488 \times 10^{-5}$ [50] consistent units is adopted as a target value for the pinched displacement under load.

The shell II consists of closed hemisphere with point loads, and a target value of 0.0924 [14] is used for the radial displacement under the loads. Results from References [9, 14, 15, 62] are used for comparison purposes.

Shell III is a variant of shell II, with a $18^{\circ}$ opening. A target value of 0.094 is adopted (see Reference [50]) for the radial displacement under the loads and the results used for comparison are those of References [12,50].

Shell IV consists of a 'partly clamped hyperbolic paraboloid' [63] and was proposed in Reference [63] as a bending-dominated test for shell elements. Two values of thickness are tested: 0.01 and $0.001 \mathrm{~m}$. For the first case, the total strain energy target has a value of $E_{n}=1.679 \times 10^{-3} \mathrm{Nm}$, and for the second case, the target value is $E_{n}=1.1013 \times 10^{-2} \mathrm{Nm}$. These values were suggested, with numerical grounds, in Reference [63].

Finally, shell V is the so-called Scordelis roof [57] and consists of a shallow cylindrical shell supported by rigid diaphragms and subject to self-weight. The monitored node is identified with a circle in Figure 10 and has a target vertical displacement of 0.3086 consistent units [57]. Results from References [1,8,9,13,14,33] are employed in the comparison. It is specially important to note that the values presented here from References [9, 14] have been re-normalized, as other target values were proposed (the value 0.3008 is quoted in Reference [8], and 0.3024 is adopted in References [9,14]) for the displacement at the mid-side.

The normalized results obtained with HIS element for the shells I-III and V are summarized in Table XII.

Figure 11 shows the convergence of the pinched node normalized displacement for shell I. Excellent behaviour of element HIS is observed. For sparse meshes, only the MITC4 shell element [61] presents better results.

For shell II, Figure 12 illustrates the good results of HIS element. The shell element of Reference [62] presents better results with sparse meshes.

The convergence curves for shell III are exposed in Figure 13, where it can be seen that element HIS is superior in terms of displacement convergence, when compared with the references.

In shell IV, the analysed value is the total strain energy. A comparison with the classical MITC4 shell element is carried out, using the values obtained in Reference [63]. Figure 14 presents the convergence curves for two values of shell thickness: 0.01 and $0.001 \mathrm{~m}$.

The observation of Figure 14 leads to some interesting conclusions: for sparse meshes, less than 16 elements on each side for a thickness of $0.01 \mathrm{~m}$ and less than 32 elements on each side for a thickness of $0.001 \mathrm{~m}$, element HIS presents a higher level of error when compared with MITC4. However, for finer meshes, the HIS element is much more accurate than MITC4 shell element. This fact is important, as this is a test to the accuracy of shell elements.

Finally, we describe the results for shell $\mathrm{V}$, where the vertical displacement at the mid-side of the free edge is monitored. The convergence of HIS element compares very favourably 

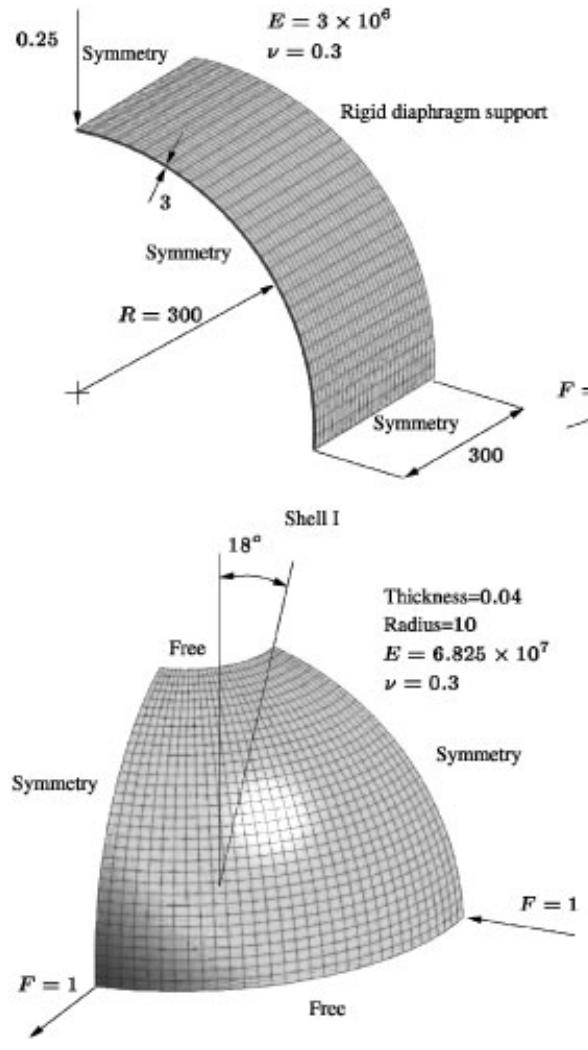

Shell III

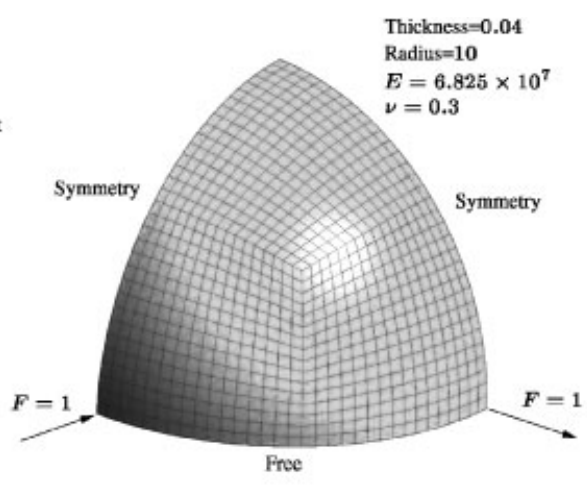

Shell II

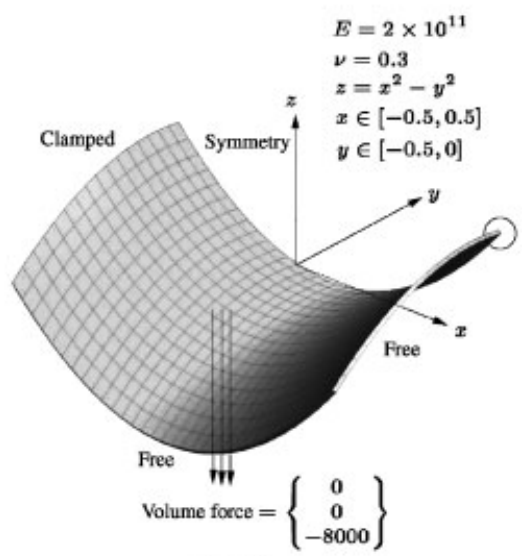

Shell IV

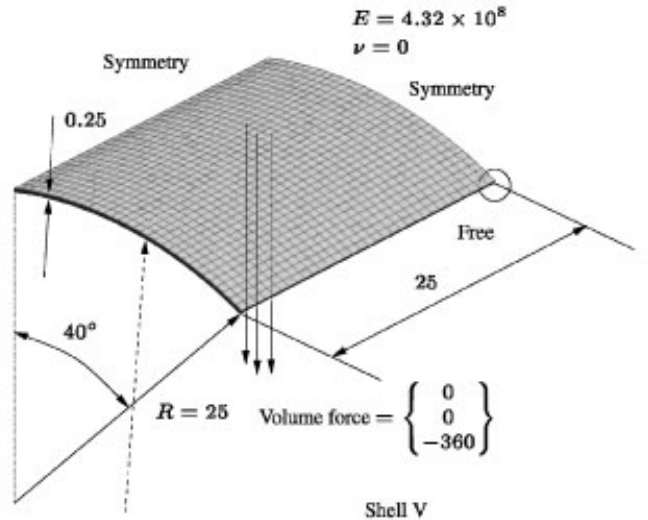

Rigid diaphragm support

Figure 10. The geometry, mesh and boundary conditions for various shell problems: shell I consists on $1 / 8$ of a pinched cylinder with diaphragm ends, shell II consists on $1 / 4$ of a closed hemisphere, shell III consists on $1 / 4$ of a open hemisphere, shell IV consists on $1 / 2$ of a clamped hyperbolic paraboloid and shell $\mathrm{V}$ consists on $1 / 4$ of a shallow cylindrical shell with rigid diaphragm support. 
Table XII. The normalized displacements, obtained with element HIS, for shells I-III and V.

\begin{tabular}{lcccc}
\hline Elements/side & Shell I & Shell II & Shell III & Shell V \\
\hline 2 & $*$ & $*$ & $*$ & 1.359 \\
4 & $*$ & 0.029 & 0.044 & 1.029 \\
5 & 0.196 & $*$ & $*$ & 1.017 \\
6 & 0.298 & $*$ & 0.361 & 1.009 \\
7 & $*$ & $*$ & $*$ & 1.004 \\
8 & 0.505 & 0.583 & 0.756 & 1.001 \\
9 & 0.606 & $*$ & $*$ & 0.998 \\
10 & 0.692 & $*$ & $*$ & 0.998 \\
16 & 0.926 & 0.978 & 0.991 & 0.992 \\
17 & 0.940 & $*$ & $*$ & $*$ \\
20 & 0.966 & $*$ & $*$ & $*$ \\
30 & 0.996 & $*$ & $*$ & $*$ \\
32 & 0.999 & 0.999 & 0.999 & 0.991 \\
\hline
\end{tabular}

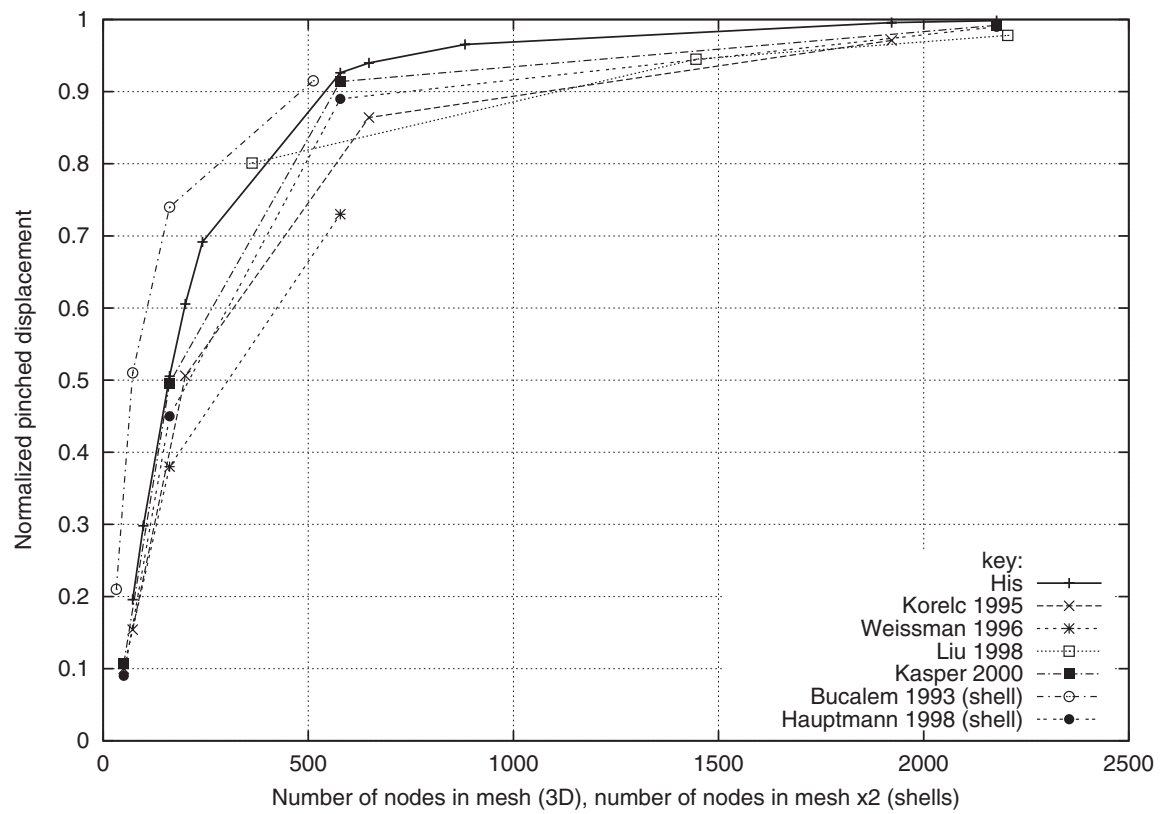

Figure 11. Shell I: convergence of normalized displacement, compared with References [4, 10, 12, 14, 50,61].

relative to the other references, as it can be observed in Figure 15. The element of Reference [9] is NUHEX-4, which in this case is superior to NUHEXIN-4, and in the case of Reference [8], the retained element is the shell element EAS7-ANS. 


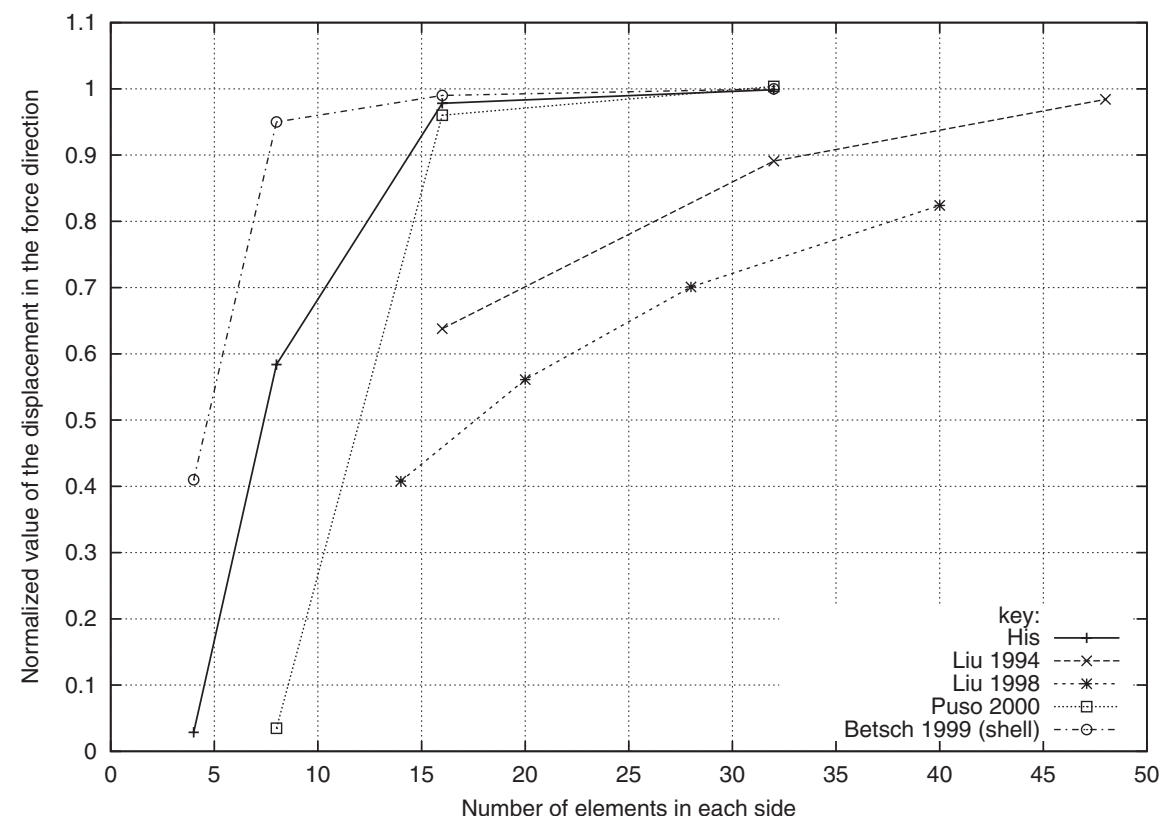

Figure 12. Shell II: convergence of normalized displacement, compared with References [9, 14, 15, 62].

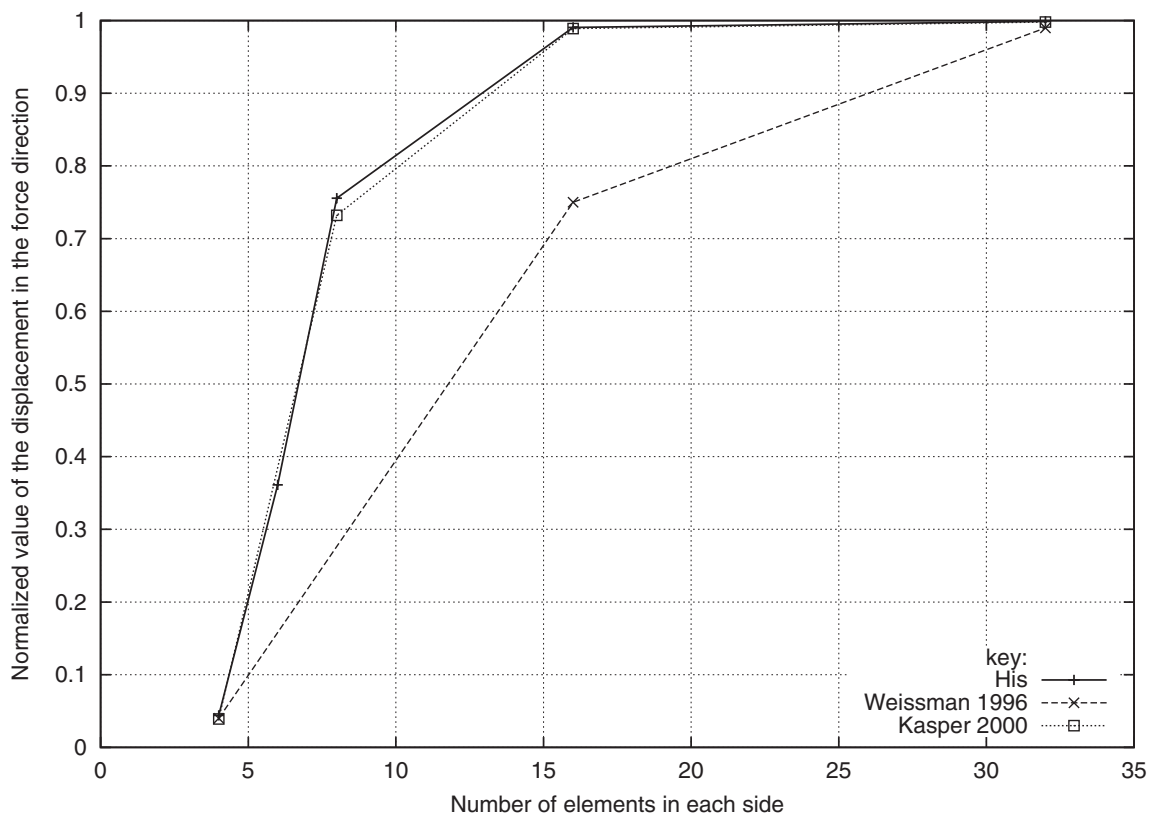

Figure 13. Shell III: convergence of normalized displacement, compared with References [12, 50]. 


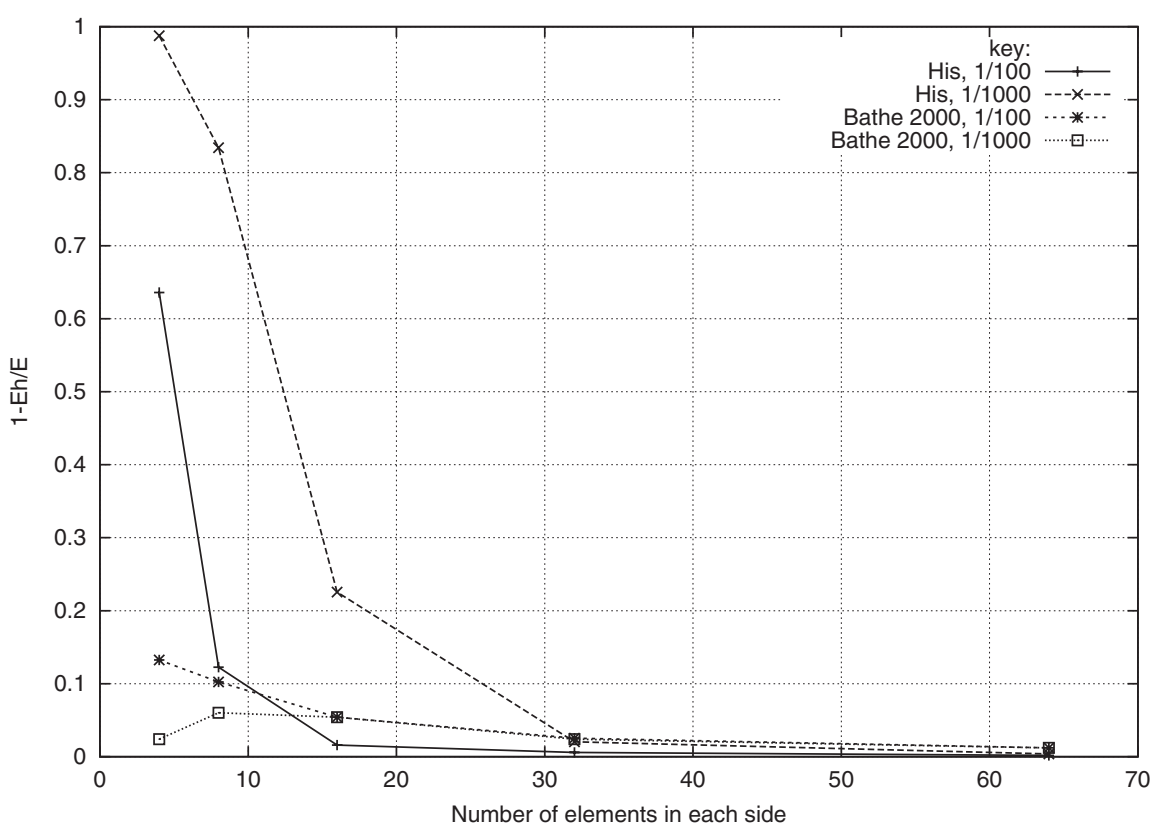

Figure 14. Shell IV: convergence of the total strain energy error with the number of elements in each side. Comparison with the results obtained with the MITC4 shell element [63].

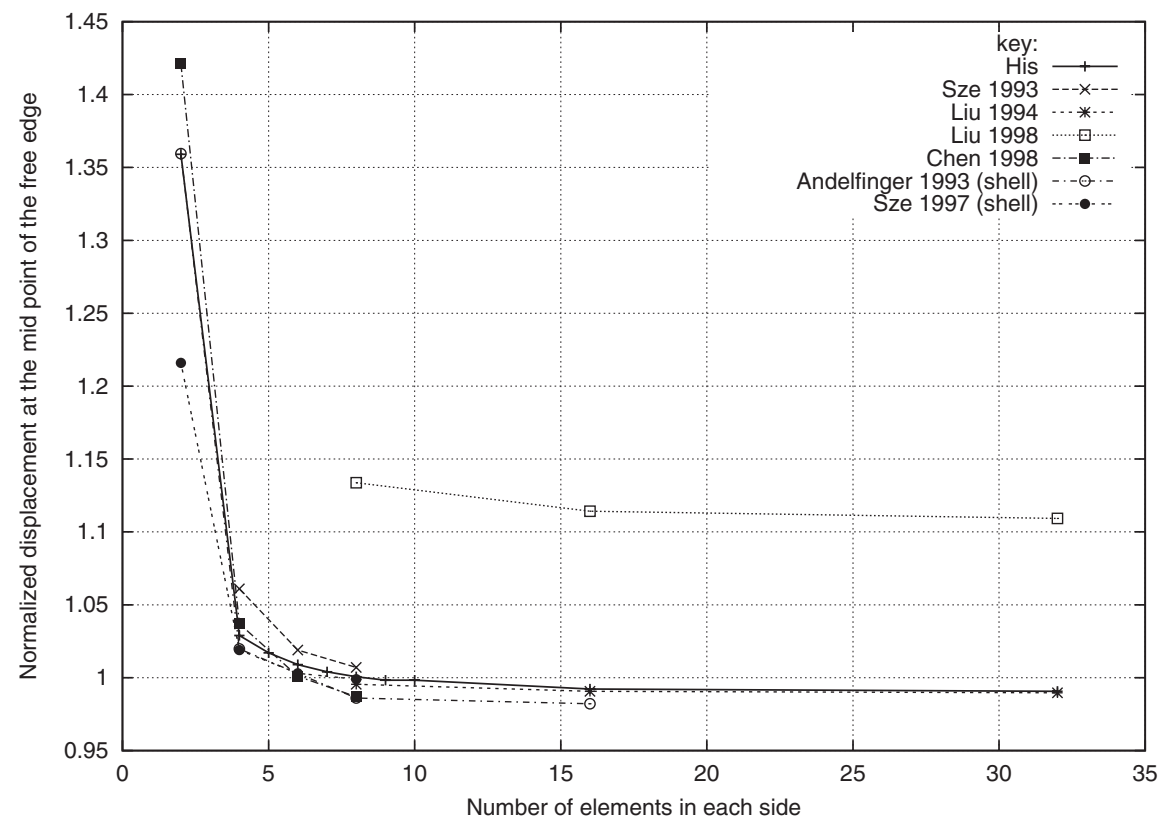

Figure 15. Shell V: convergence of normalized displacement, compared with References $[1,8,9,13,14,33]$. 


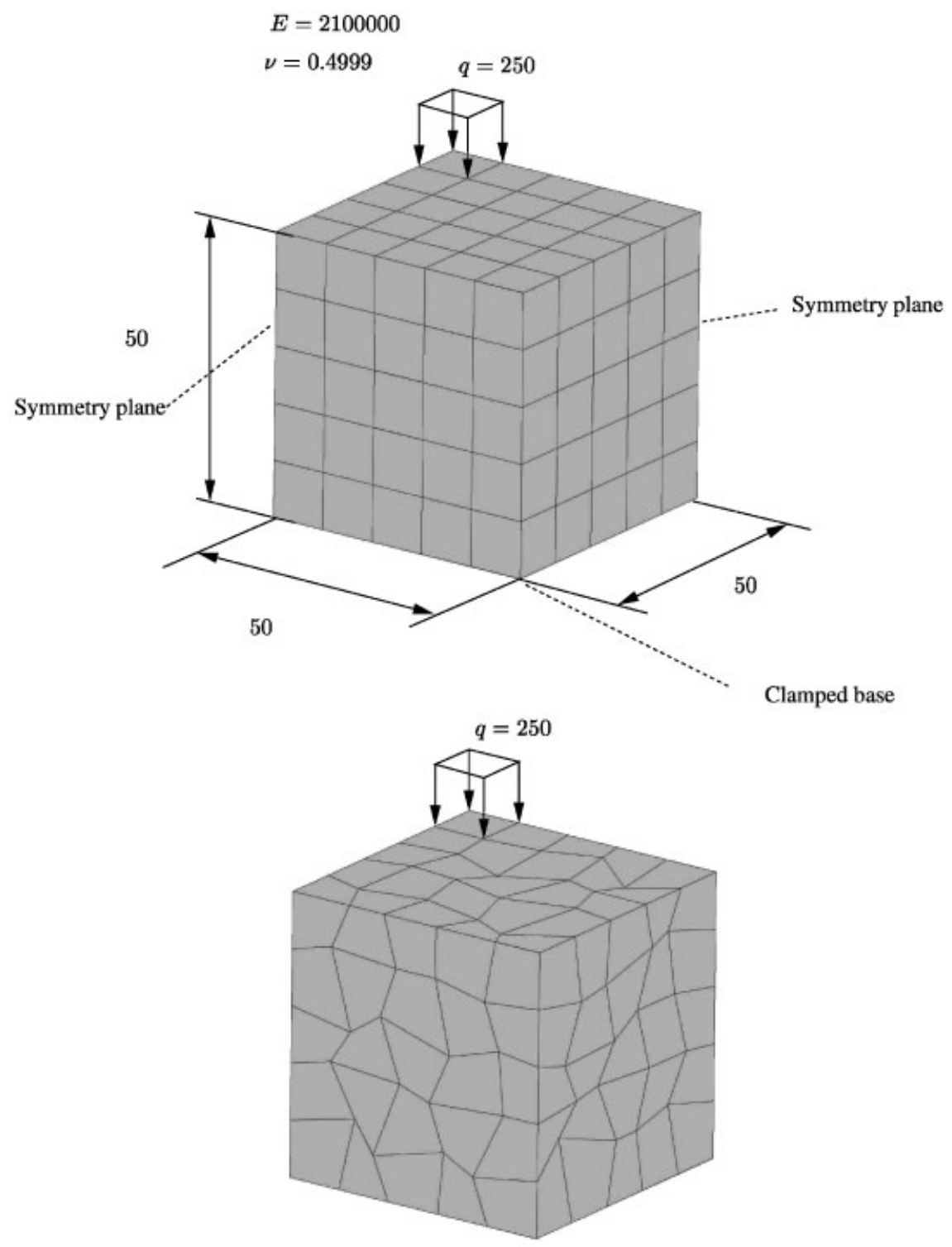

Figure 16. Near-incompressible block: geometry, boundary conditions and the two tested meshes.

3.2.4. Near-incompressible block. This example consists of regular block with a height of 50 and a side of 100 (see References $[8,10]$ ). The elastic properties of the block are $E=210000$ and $v=0.4999$. The block is loaded in its centre by a uniform distributed load acting on a square area of $20 \times 20$. Only one-fourth of the block is actually meshed. Two types of mesh are tested: a regular one and a distorted one. These two meshes both with $5 \times 5 \times 5$ elements, along with boundary conditions are presented in Figure 16. The vertical displacement at the 
Table XIII. Near-incompressible regular block, displacement at the centre of the block for the distorted and non-distorted meshes.

\begin{tabular}{lcc}
\hline Element & $\begin{array}{c}\text { Displacement, } \\
\text { non-distorted mesh }\end{array}$ & $\begin{array}{c}\text { Displacement, } \\
\text { distorted mesh }\end{array}$ \\
\hline HIS & 0.01921 & 0.01920 \\
QM1/E12 [10] & 0.01892 & 0.01840 \\
QS/E9 [10] & 0.01910 & 0.01887 \\
QSN/E9 [10] & 0.01910 & 0.01885 \\
3D.EAS-30 [8] & 0.01905 & 0.01852 \\
\hline
\end{tabular}

central point of the block is presented in Table XIII where the results from References $[8,10]$ are also presented. The proposed element gives practically the same answer with both meshes. Other formulations give stiffer results and suffer from mesh sensitivity. The elements QS/E9 and QSN/E9 are described in Reference [10] and the element QM1/E12 is the element first proposed in Reference [19]. The last element in Table XIII is proposed in Reference [1] and contains 30 internal variables.

\subsection{Non-linear problems}

3.3.1. Pure bending of a cantilever beam. This classical geometrically non-linear solid mechanics problem (described in Reference [64]) consists of an elastic cantilever beam subject to a bending moment at one end and clamped at the opposite end. The moment intensity is gradually increased until the beam middle surface forms a perfect cylinder. This problem has been solved numerically in several papers. Recently, in References $[65,66]$ a simulation of this test was carried out in the context of validating procedures for finite rotation of shell elements. The present HIS solid model has no difficulty in dealing with this problem, as long as a deformation-dependent loading is introduced to apply the moment. The geometry and boundary conditions exposed in References $[65,66]$ are here reproduced.

For the application of the end moment, $M$, the following formula is employed:

$$
M=\frac{2 E I \pi n}{L}
$$

where $E$ represents the elasticity modulus, $I$ represents the second-order moment of the crosssection relative to the bending moment direction and $L$ represents the beam length. The integer $n$ represents the number of turns of the beam (which is 1 in the present case). The Poisson coefficient is $v=0$ and the elasticity modulus has a value of $E=1.2 \times 10^{7}$ consistent units.

Figure 17 presents the 25 element mesh and the boundary conditions in the non-deformed case for $\theta=0$, and also the final deformed mesh, for $\theta=2 \pi$. In Reference [65] the same problem was analysed with a $25 \times 2$ shell element mesh.

From the observation of Figure 17 it is possible to conclude that the deformed mesh is representing a nearly perfect circular ring. This remarkable accuracy is accomplished without the use of rotation variables and inherent update complexities (see Reference [65] for an account of such procedures). 

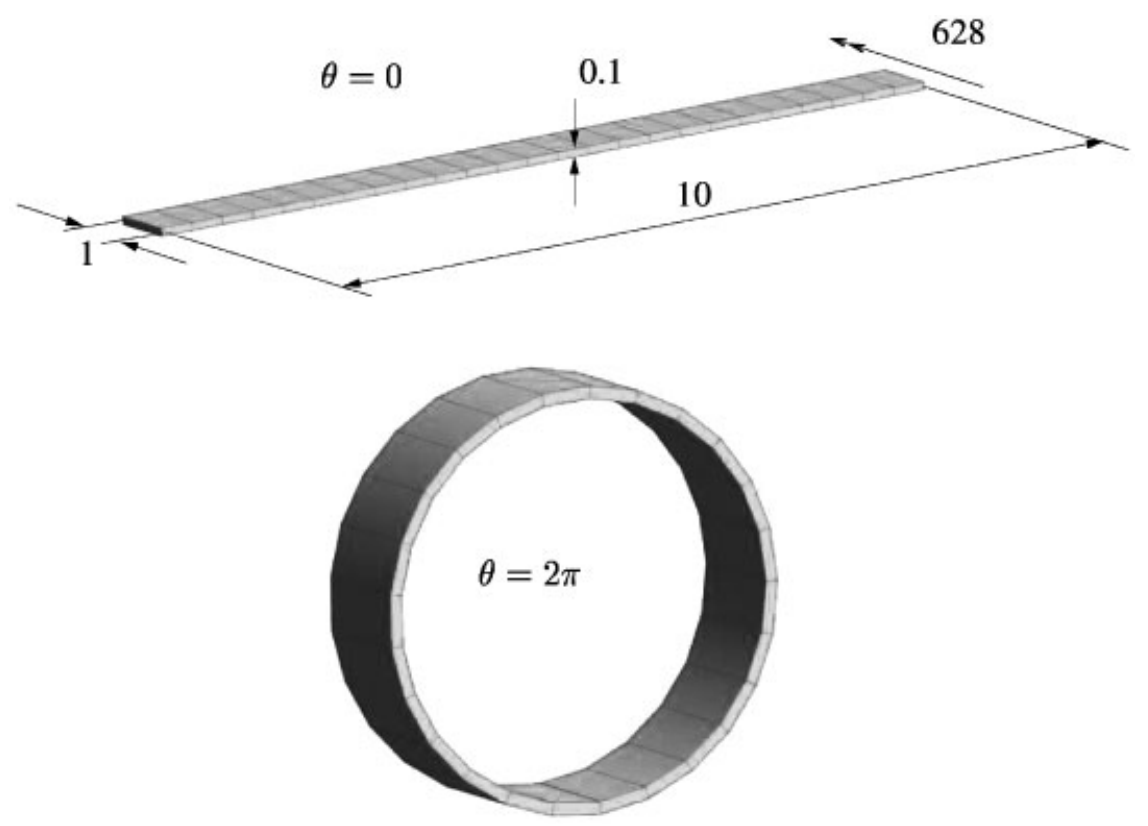

Figure 17. The non-deformed cantilever beam mesh, and the deformed mesh for a moment of 628 consistent units, corresponding to a rotation of $2 \pi$ radians.

3.3.2. Thin plate ring. This problem was first considered by Basar and Ding [67] to test formulations for finite shell rotations. Several other authors have also studied this problem $[60,68-70]$. The test consists in the pulling of a thin circular ring containing a radial cut. The pulling is carried out resorting to a distributed load with fixed direction, applied on a edge. The opposite edge is clamped.

The ring is considered elastic with an elasticity modulus of $E=2.1 \times 10^{10}$ and a Poisson coefficient of $v=0$. The geometry, mesh, and boundary conditions are represented in Figure 18 . The mesh contains $6 \times 30 \times 1$ elements in agreement with Reference [70], where three types of shell elements (hybrid strain, hybrid stress and enhanced strain) were tested with similar results for this mesh.

For comparison purposes, Reference [70] is adopted. Figure 19 shows the load-displacement curves. Excellent agreement is obtained between Reference [70] results and the present paper ones.

3.3.3. Hinged cylindrical shell under a central point load. This test has been considered by Sabir and Lock [71] and latter by Ramm [56], and it is a popular test (a non-exhaustive list of other references where this test has been presented and studied is $[3,66,72-76]$. The test is useful to verify both the non-linear finite element formulation and the path following algorithms.

It consists of a pinched shallow cylinder with hinged longitudinal edges and free curved edges, as depicted in Figure 20. Owing to symmetry, only one-fourth of the model is actually 

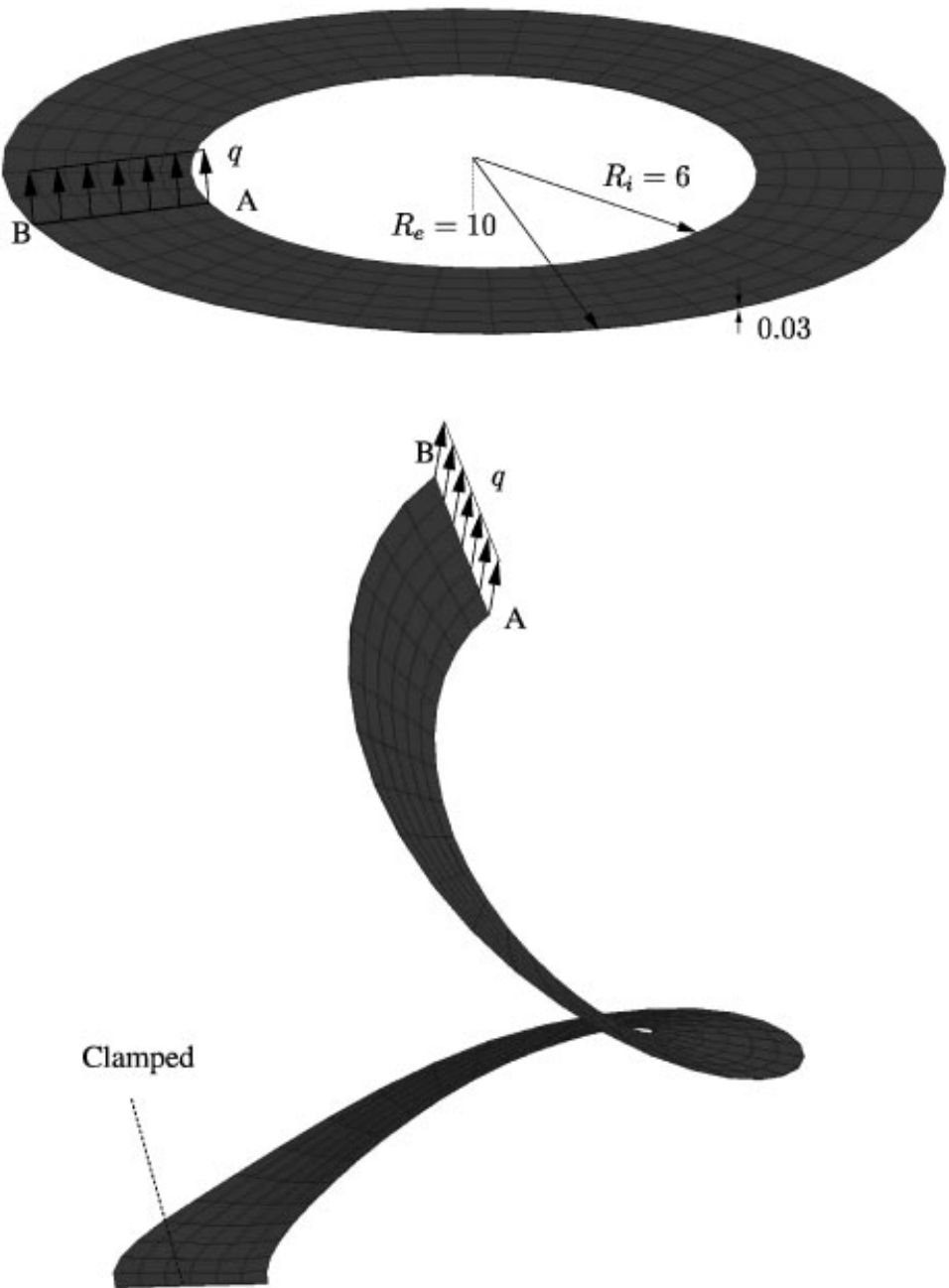

Figure 18. Thin plate ring: geometry, boundary conditions and deformed mesh for a load $q=2000$.

meshed. A thickness of $6.35 \mathrm{~mm}$ is known to induce both snap-through and snap-back behaviour, as detailed in Reference [72]. In this paper, a standard spherical arc-length procedure is adopted which is analogous to the one described in Reference [56]. In terms of comparison of results, two references are used: the Abaqus examples manual [74] where the reduced integration shell element S4R was used with a $6 \times 6$ mesh and the Reference [56] where a $2 \times 2$ mesh of bi-cubic 16 noded elements was employed. The vertical displacement of points $\mathrm{A}$ and $\mathrm{B}$ identified in Figure 20 is monitored.

In this paper a $6 \times 6 \times 2$ mesh is used. There is a need of employing 2 elements along the thickness to ensure symmetry in the hinged boundary relative to the mesh, which was found to be a crucial aspect in terms of accuracy. 


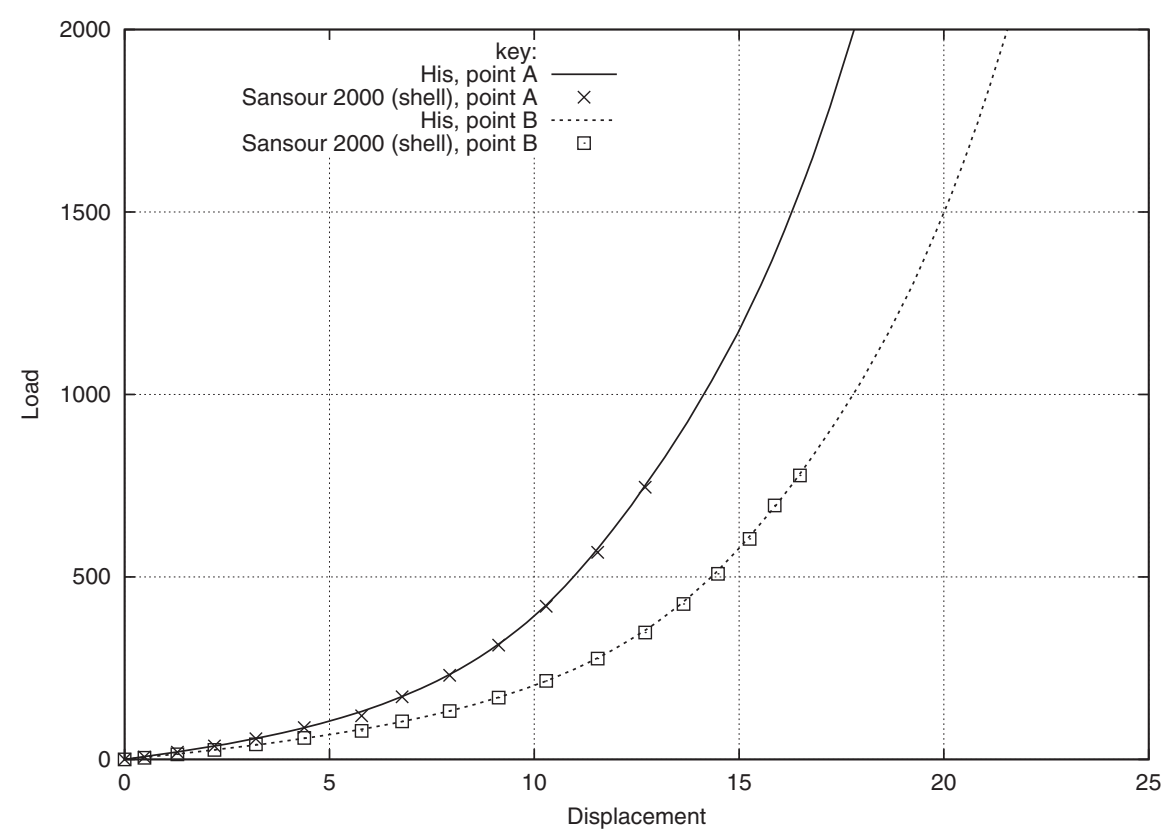

Figure 19. Thin plate ring: load-displacement curves, for points A and B, identified in Figure 18, comparison with Reference [70].

The adopted material is considered elastic with an elasticity modulus $E=3.103 \times 10^{3} \mathrm{~N} / \mathrm{mm}^{2}$ and the Poisson coefficient $v=0.3$.

The load-deflection curves for points A and B are depicted in Figure 21 and excellent agreement with both the low-order S4R element and the high-order 16 node element is observed.

3.3.4. Pinched cylinder with free edges. This example has been described and studied by various other authors $[60,70,73,77]$ and constitutes a difficult test for finite element formulations, combining bending and membrane effects. The example consists of a cylinder shell with open ends which is pulled in two diametrically opposite points through the application of point forces. The geometry, mesh and boundary conditions for one-eighth of the model are presented in Figure 22. Points A, B and C in Figure 22 are monitored in terms of absolute radial displacement. The material is considered elastic, with elasticity modulus $E=10.5 \times 10^{6}$ consistent units and Poisson coefficient $v=0.3125$, in agreement with the above references. A mesh with 16 elements along the circumferential direction, 8 elements along the longitudinal direction and 2 elements along the thickness is adopted. This mesh has been used in Reference [77] within exactly same context.

In Reference [77], the analysis was carried out in 140 steps. The present analysis takes only 50 steps with a much higher load (200 instead of 38 consistent units). The absolute radial displacement for points $\mathrm{A}-\mathrm{C}$ is compared with the values obtained in Reference [77]. Figure 23 shows this comparison. Very good agreement is obtained for the branches A and B. 


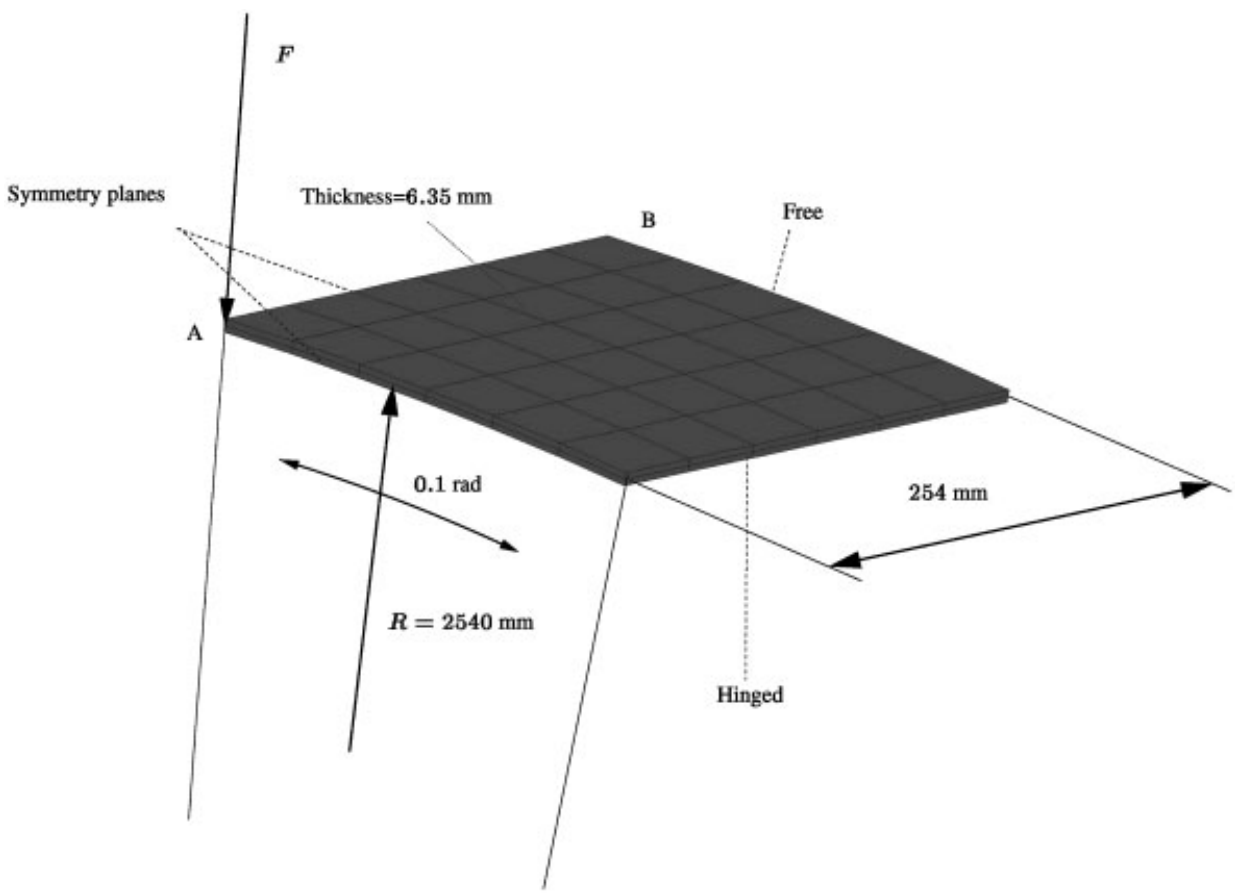

Figure 20. Hinged cylindrical shell: geometry, mesh and boundary conditions for one-fourth of the shell.

Branch $\mathrm{C}$ is slightly different. It is, however, important to note that Reference [78] presents a branch $\mathrm{C}$ similar to the one obtained in the present paper.

3.3.5. Open hemisphere loaded by pinching forces. This example is a non-linear counterpart of the linear elastic open hemisphere example illustrated in Figure 10. The shell suffers large displacements but strains and rotations are relatively small (see Reference [65]). The test is very popular and has been studied in References $[4,5,60,65,69,70,73,77,79-81]$. The Figure 24 shows the non-deformed mesh and a deformed mesh of the total hemisphere model. The nodes $\mathrm{A}$ and $\mathrm{B}$ identified in the figure are monitored in terms of absolute radial displacement. The material is considered elastic with an elasticity modulus $E=6.825 \times 10^{7}$ and a Poisson coefficient of $v=0.3$. The radius is 10 consistent units and the thickness value is 0.04. Only one-fourth of the model is actually meshed with two types of meshes: $16 \times 16 \times 1$ elements and $24 \times 24 \times 1$ elements. Most authors use a $16 \times 16 \times 1$ mesh, but Reference [69] shows results for $24 \times 24 \times 1$ mesh. The Reference [4] also proposes a finer $32 \times 32 \times 1$ mesh.

A comparison, in terms of load-displacement curves, between the present formulation and the ones of References $[65,69,70]$ is presented in Figure 25. Very good agreement is observed for the $24 \times 24 \times 1$ element mesh are obtained between the present formulation and both of the above references. It is clear that a much higher level of load can be achieved by the HIS formulation. 


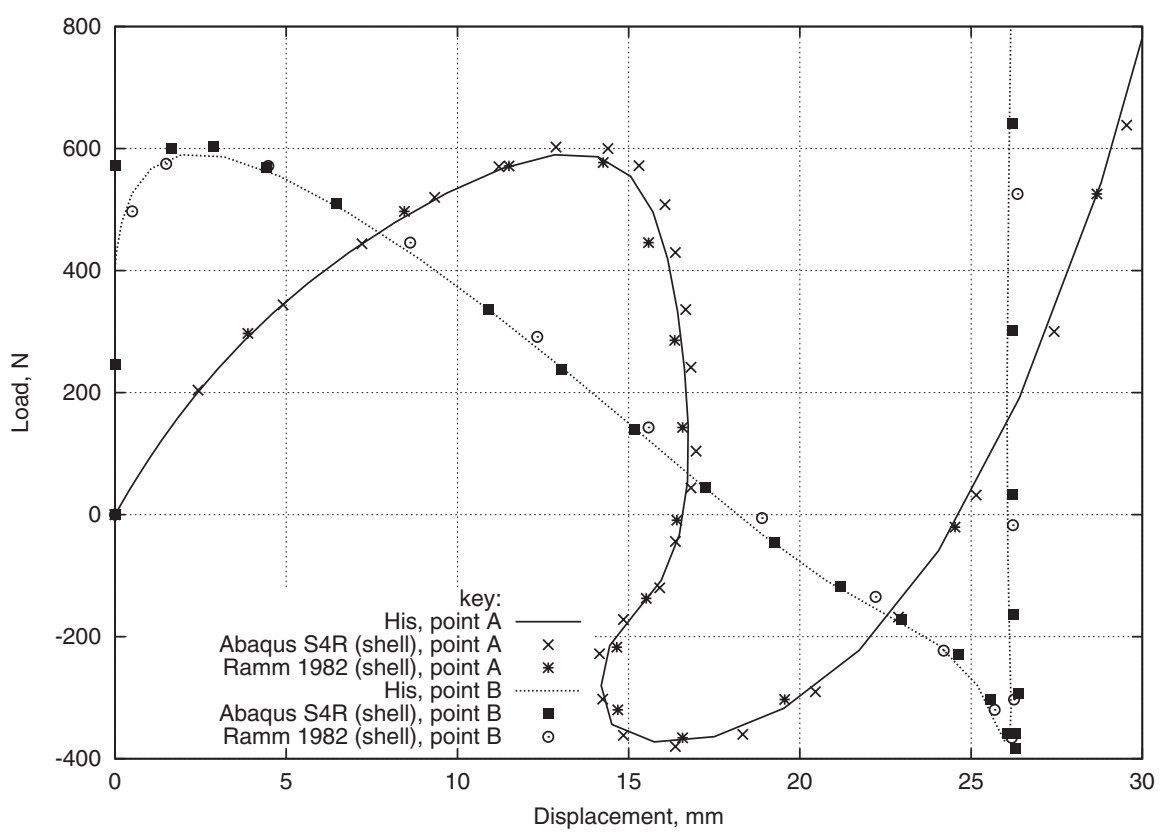

Figure 21. Hinged cylindrical shell: load-deflection curve for points $\mathrm{A}$ and $\mathrm{B}$, comparison with References $[74,56]$.

3.3.6. Pinched cylinder with end diaphragms. This test was considered, with a distinct geometry, by Simo and Kennedy [82]. The test is a geometrical and material non-linear version of the linear pinched cylinder test which can be observed in Figure 10. It involves very large displacement and strains, and is difficult for certain finite element formulations to achieve Newton-Raphson convergence at the last stages of the analysis, as mesh deformation is present. Several other authors have studied this test (see References $[38,70,83,84])$. The geometry is the one previously identified for the linear case, with a total length of 600 , a radius of 300 and a thickness of 3 consistent units. Other authors have presented distinct dimensions [70]. The elasticity modulus $E=3000$ consistent units and the Poisson coefficient $v=0.3$. The hardening law is presented in Equation (32) where $\sigma_{y}$ represents the yield stress and $\varepsilon_{p}$ represents the accumulated effective plastic strain. Other references, like [83], propose distinct hardening laws. The hardening law agrees with References [38, 84].

$$
\sigma_{y}=24.3+300 \varepsilon_{p}
$$

The adopted mesh contains only $16 \times 16 \times 1$ elements and it has been used in Reference [84]. A much more refined mesh is presented in Reference [84], with $32 \times 32 \times 1$ elements. A total pinched displacement of 300 consistent units is applied in the present analysis, in contrast with the values 250 in Reference [38] and 280 in Reference [84]. This imposed displacement is coincident with the initial radius, which undoubtedly induces a severe case of mesh deformation. Two types of end diaphragms are tested, corresponding to a 'hard support' 

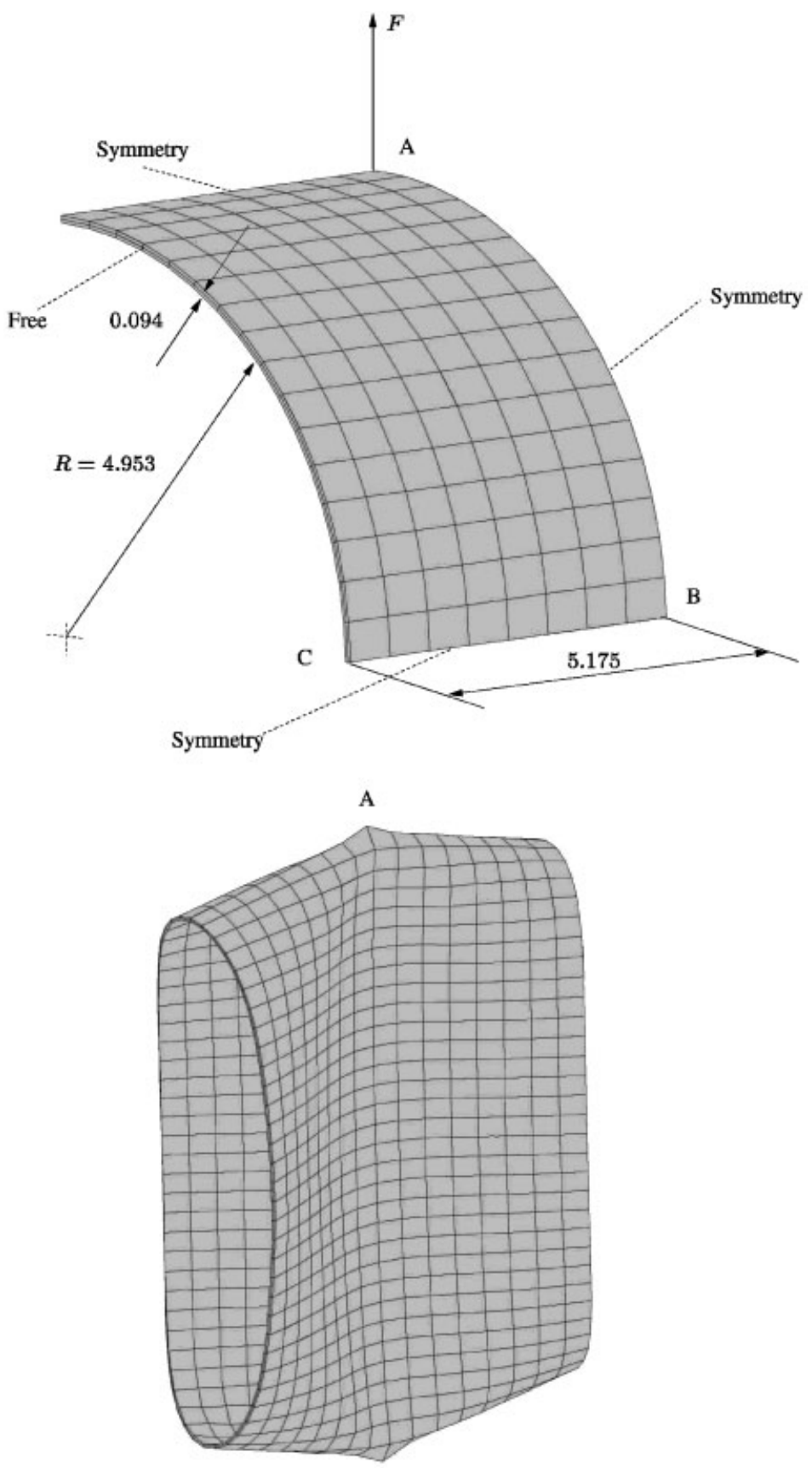

A

Figure 22. Pinched cylinder with free edges: geometry, mesh and boundary conditions for one eighth of the model, and final deformed mesh. 


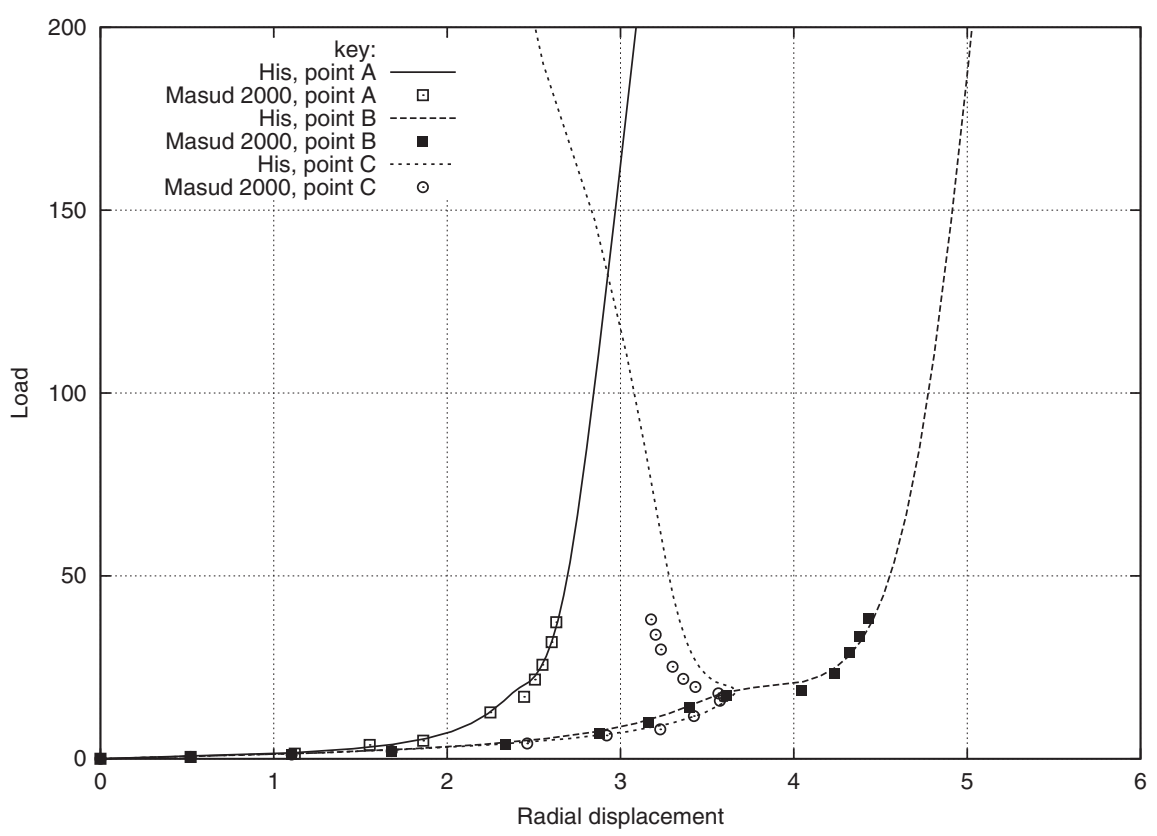

Figure 23. Pinched cylinder with free edges: comparison of loaddisplacement curves with Reference [77].

and 'soft support', where in the second case, rotation of the diaphragm is allowed (see Reference [38] for further details). In the present work, the hard support is identified as case A and the soft support is identified as case B.

The non-deformed mesh, and the final deformed meshes for cases A and B are all shown in Figure 26.

The load-displacement curves for both cases are compared with the results obtained in Reference [84] and with the 5-parameter shell element of Reference [84] where only case A was tested. Figure 27 presents the results. Very good agreement in the results is verified between the present formulation and both shell elements, with HIS element presenting a slightly more soft behaviour in the membrane dominated part of the curve (which corresponds to higher displacements). Note that the snap-through situation at the middle of the analysis for case B, verified both in Reference [83] and in Reference [84] is also perfectly well represented by the HIS element.

3.3.7. Square plate under pressure. This test consists in the analysis of a simply supported square plate with elasto-plastic behaviour. The plate is subjected to a deformation-dependent uniform pressure. It has been studied in References [2, 6, 62, 84, 85] and combines the bending efforts at the first stages of the analysis and membrane efforts at the latter stages. The plate has dimensions $508 \times 508 \times 2.54$. The elasticity modulus $E$ has a value of $6.9 \times 10^{4}$ consistent units and the Poisson coefficient $v$ has a value of 0.3 . No hardening is present and the yield stress $\sigma_{y}$ has a value of 248 consistent units. One-fourth of the geometry is discretized 


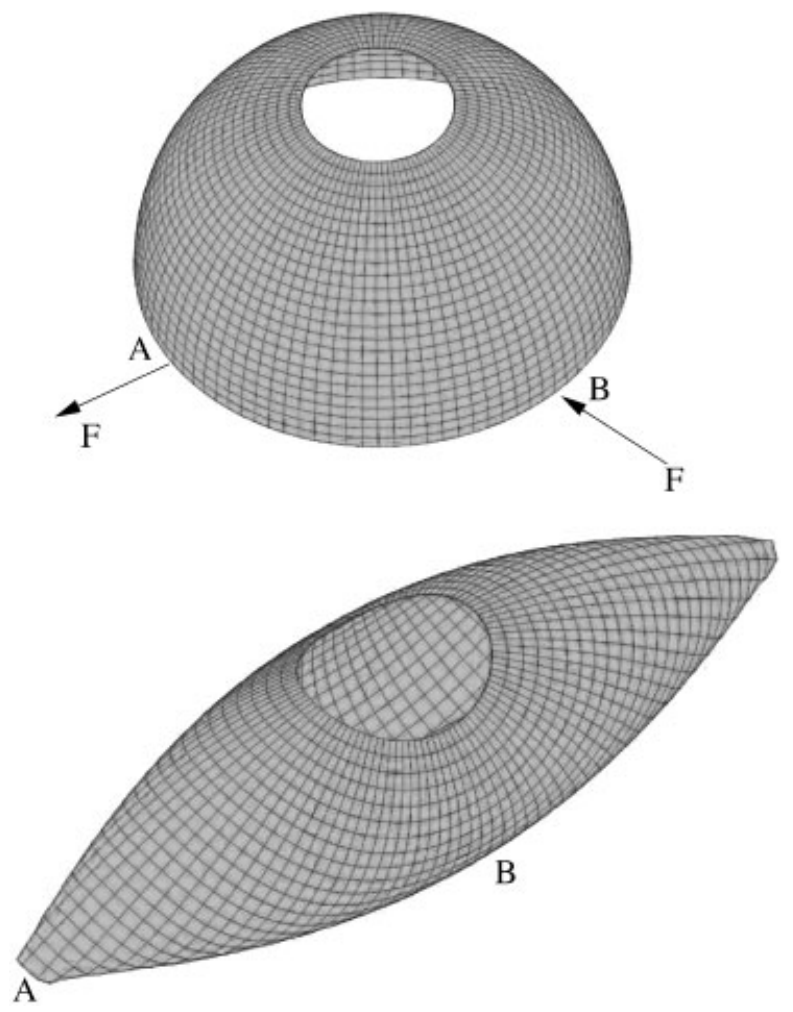

Figure 24. The non-deformed and a deformed configuration of the open hemisphere loaded by pinching forces.

with $24 \times 24 \times 1$ elements, which agrees with Reference [62]. This corresponds to the use of 2 integration points in the thickness direction. For comparison, Reference [6] uses a mesh of $32 \times 32 \times 1$ solid-shell elements with 6 integration points in the thickness direction. In contrast with these meshes, Reference [84] adopts a non-uniform mesh, refined in the plate corners.

The non-deformed mesh and the deformed mesh for a pressure value of $p=4.33$ consistent units are displayed in Figure 28. It is clear from this figure that, at this high value of pressure, wrinkles start to form at the mid-sides of the plate. This effect has never, in the authors knowledge, been noticed in other references, as the pressure never reached this high value. Both the corners, the wrinkles and the top of the deformed plate are fully plastified.

In terms of pressure-displacement curves, a comparison is carried out with the values obtained in References [62,84]. The node at the plate's centre is monitored. The values of Reference [84] correspond to the 6-parameter model. Figure 29 presents the various curves. Very close agreement between the present analysis and the one in Reference [62] is observed. It is also clear that Reference [84] presents stiffer results. 


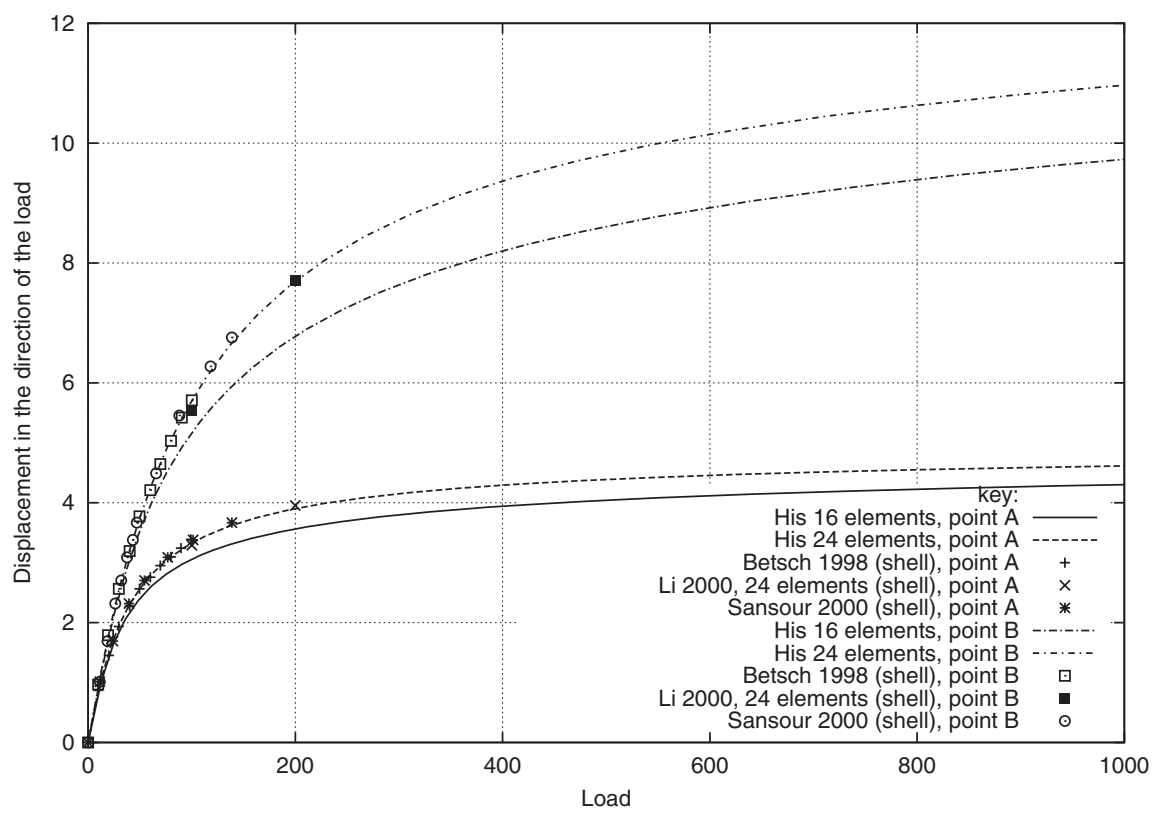

Figure 25. Open hemisphere: comparison of load-displacement curves with References $[65,69,70]$.

3.3.8. Elasto-plastic tension test. This test has been considered by Simo and co-workers in Reference [18] in the context of validation of algorithms for finite strain plasticity and, with a distinct mesh disposition, in Reference [18], in the context of testing enhanced strain finite elements.

The problem consists in the pulling of a bar up to a displacement of 7 consistent units. One-eighth of the bar is represented in Figure 30 along with the final distribution of the effective plastic strain. The material considered is elasto-plastic with the following hardening rule:

$$
\sigma_{y}=0.45+0.12924 \varepsilon_{p}+(0.715-0.45)\left(1-\mathrm{e}^{-16.93 \varepsilon_{p}}\right)
$$

The elasticity modulus $E$ value is 206.9 consistent units and the Poisson coefficient $v$ value is 0.29 .

It is clear from the observation of Figure 30 that an imperfection in the radius is introduced (see also References $[18,54]$ ) to ensure that necking occurs. A mesh of 960 elements is adopted, disposed according to the Figure 30.

The tension force is monitored and compared with the results obtained with a 9 internal variable element from Reference [18]. In Figure 31 it is visible that the standard 9 internal variable element does not present a locking-free behaviour, as verified, for example, in Reference [19] where a 12 internal variable node was proposed.

Figure 31 also shows the excellent Newton-Raphson convergence behaviour, as the line segments in the curve relative to the proposed element represent time-step increments. 


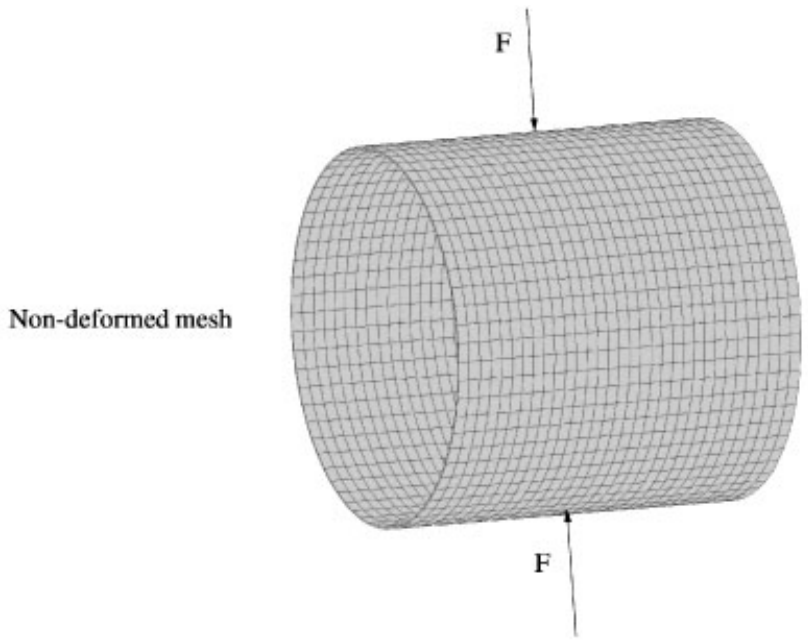

Deformed mesh, case A

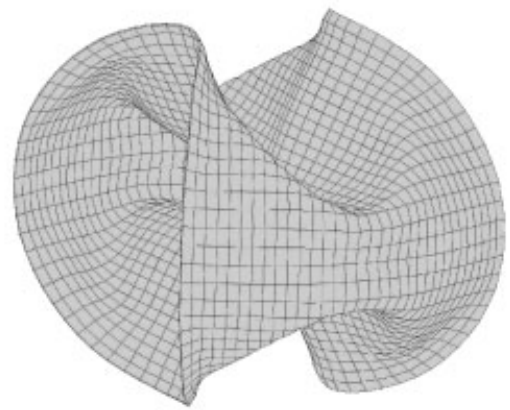

Deformed mesh, case B

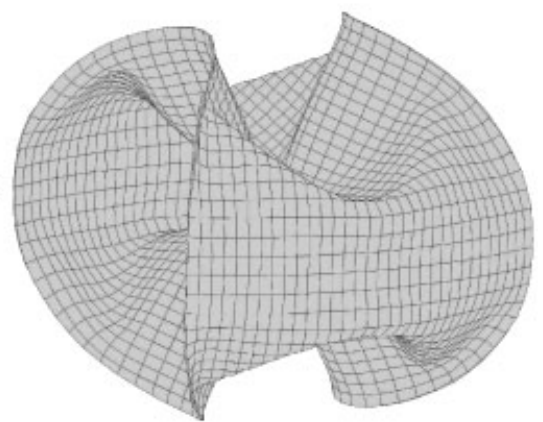

Figure 26. Pinched cylinder: non-deformed mesh and deformed meshes for cases A and B. 


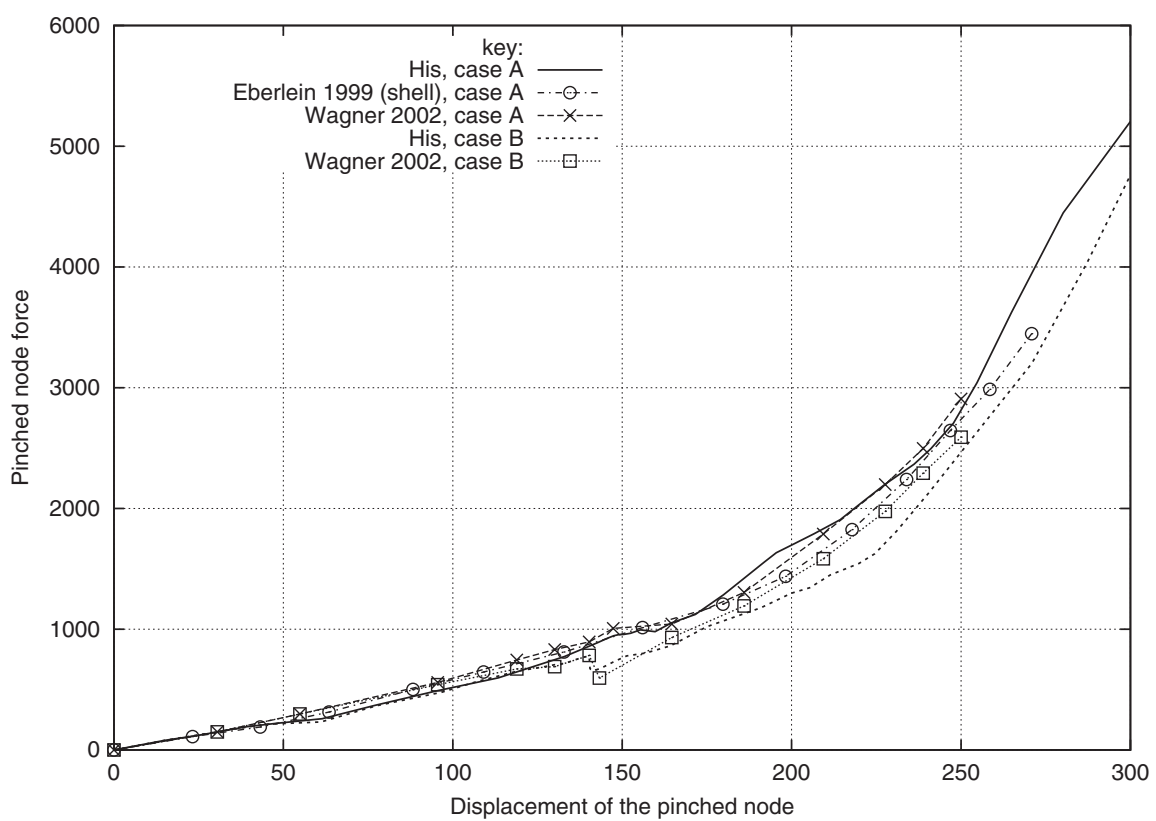

Figure 27. Pinched cylinder: comparison of the load-displacement evolution with References [38, 84].

3.3.9. Silicone rubber tension test-comparison with experimental results. This example consists of the analysis of a normalized tension test, which is validated experimentally, of a silicone rubber specimen. The considered material is an incompressible Yeoh model (see Reference [47]), whose deviatoric strain energy function is given by

$$
W=c_{1}\left(\bar{I}_{1}-3\right)+c_{2}\left(\bar{I}_{1}-3\right)^{2}+c_{3}\left(\bar{I}_{1}-3\right)^{3}
$$

where the material property values are $c_{1}=0.2528327 \mathrm{MPa}, c_{2}=0.1139638 \mathrm{MPa}$ and $c_{3}=$ $-0.01061744 \mathrm{MPa}$. The term $\bar{I}_{1}$ in (34) is the second deviatoric invariant. The non-deformed and deformed meshes for the specimen are presented in Figure 32.

The stress-strain curve for the experimental (the experiment was carried out by Prof. António Torres Marques at INEGI, laboratories, Portugal), theoretical and numerical cases is represented in Figure 33.

3.3.10. Indentation of a near-incompressible block-the need for hourglass stabilization. This last example is the only one where the stabilizing parameter takes a value distinct from 0 . This problem has been dealt in the 2D case in Reference [22], where hourglass was detected both in near-incompressible elastic and elasto-plastic situations. The near-incompressible situation is here reproduced for the $3 \mathrm{D}$ case. The test consists in the partial $(20 \%)$ indentation of a block with dimensions $2 \times 1 \times 1$. A regular mesh of $10 \times 10 \times 2$ elements is adopted. Figure 34 shows the geometry and boundary conditions for the block. Figure 35 illustrates the occurrence of hourglass. 

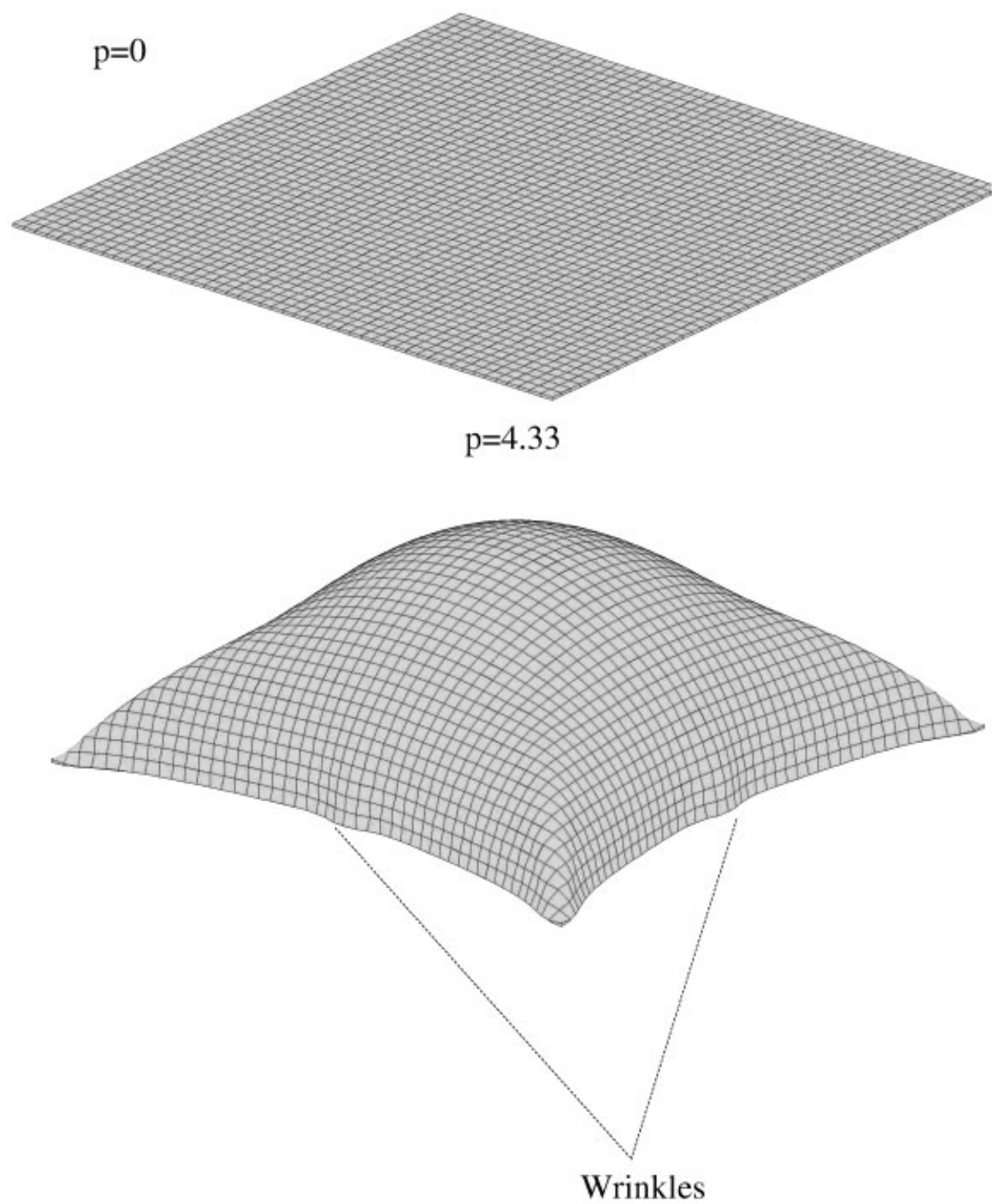

Figure 28. Square plate under pressure: non-deformed and deformed mesh $(p=4.33)$, where the birth of wrinkles at the mid-sides of the plate is visible.

For the non-stabilized case, the analysis stops at an indentation of 0.02414 , due to the presence of instabilities. These instabilities are a well-known defect of enhanced strain elements (see References [19, 20, 22, 86]).

The hourglass patterns visible in Figure 35 for the non-stabilized case can be completely eliminated with the use of the stabilizing term. If a value of $r=1.5 E /(1+v)$ is used for the penalty parameter, then the analysis is carried out until the end and without any apparent 


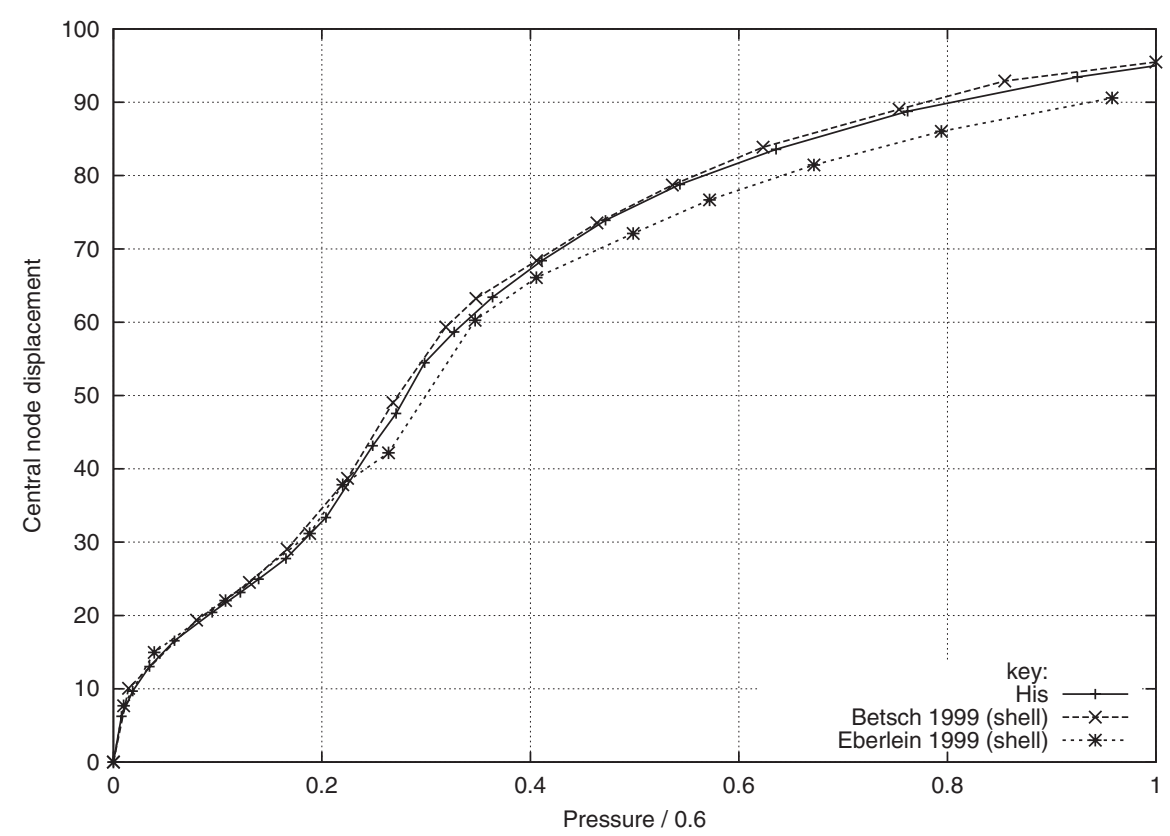

Figure 29. Square plate under pressure: pressure-displacement curves. Comparison with References $[62,84]$.

hourglass patterns. Owing to the premature abortion of the unstable case, reactions for a low order mixed $u-p$ element, implemented by the first author of this paper, are used for comparison with the stabilized case. Figure 36 presents the reaction curves for the HIS element and the $u-p$ element.

\section{CONCLUSIONS}

The proposed low-order HIS solid element, which is a general-purpose element, can be applied, without modifications, to various solid mechanics problems, from beam, plate and shell analysis to bulk forming and finite strain elasto-plastic analysis. Even complex implicit gradient models for modelling strain softening behaviour have been implemented with success with a variant of the present element (see Reference [51]).

In terms of linear elastic accuracy, if mesh is regular, the element behaviour is very good as the tests performed in this paper illustrate. There are 3D mixed or hybrid elements (see References $[7,12,13,35]$ ) which present higher degree of accuracy in certain linear elastic problems, but at the cost of lack of generality in the range of possible applications.

For non-linear analysis, the element has a good behaviour, with results extremely close to the best shell elements in a number of shell problems and also presents very accurate results in 3D solid analysis. The Newton-Raphson convergence characteristics were excellent and in the non-linear problems presented, the analysis could be easily extended to much higher values of loading, as it became clear. 

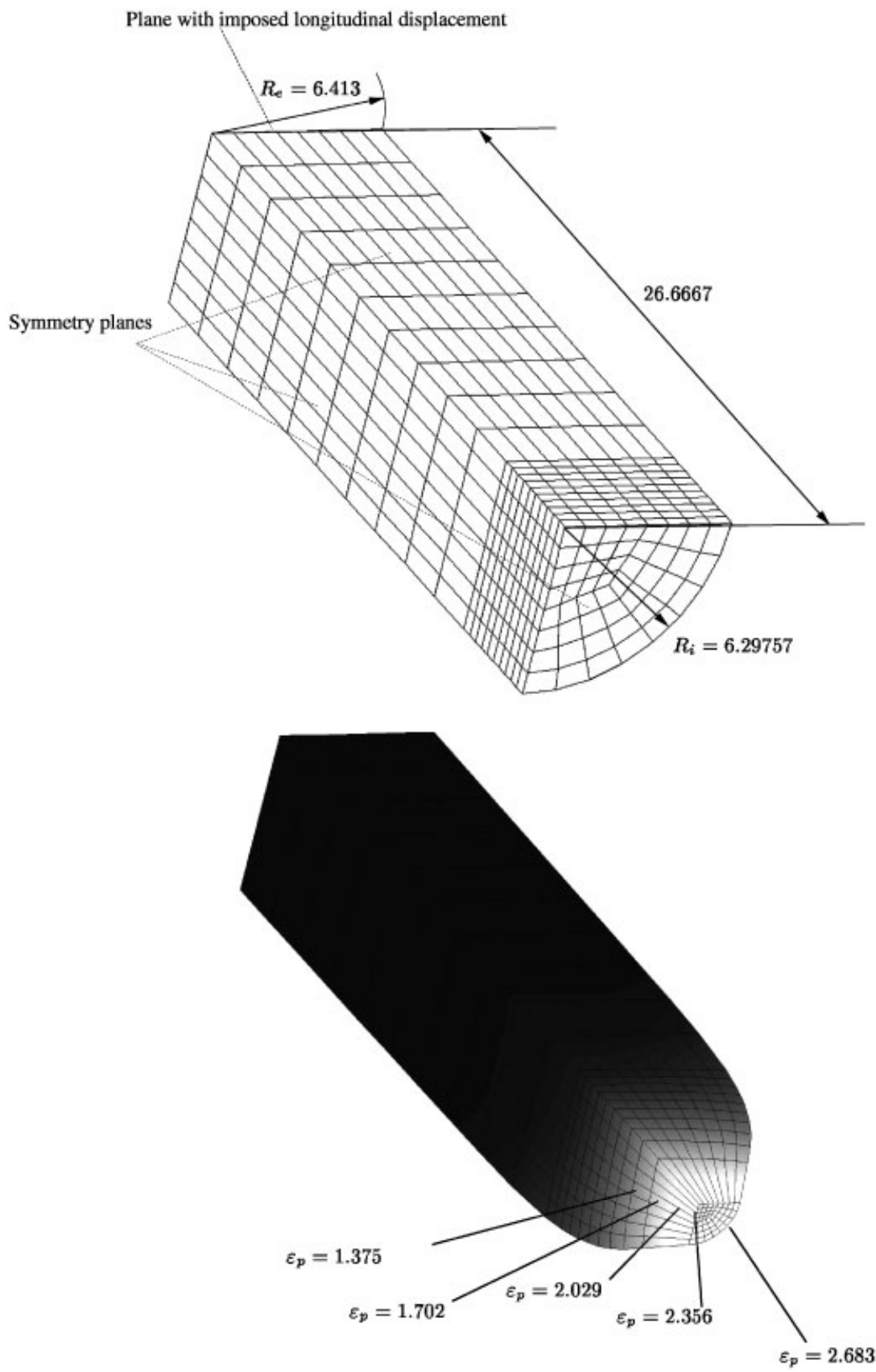

Figure 30. The geometry and boundary conditions of the tension bar and the final deformed mesh with effective plastic strain contour plot. 


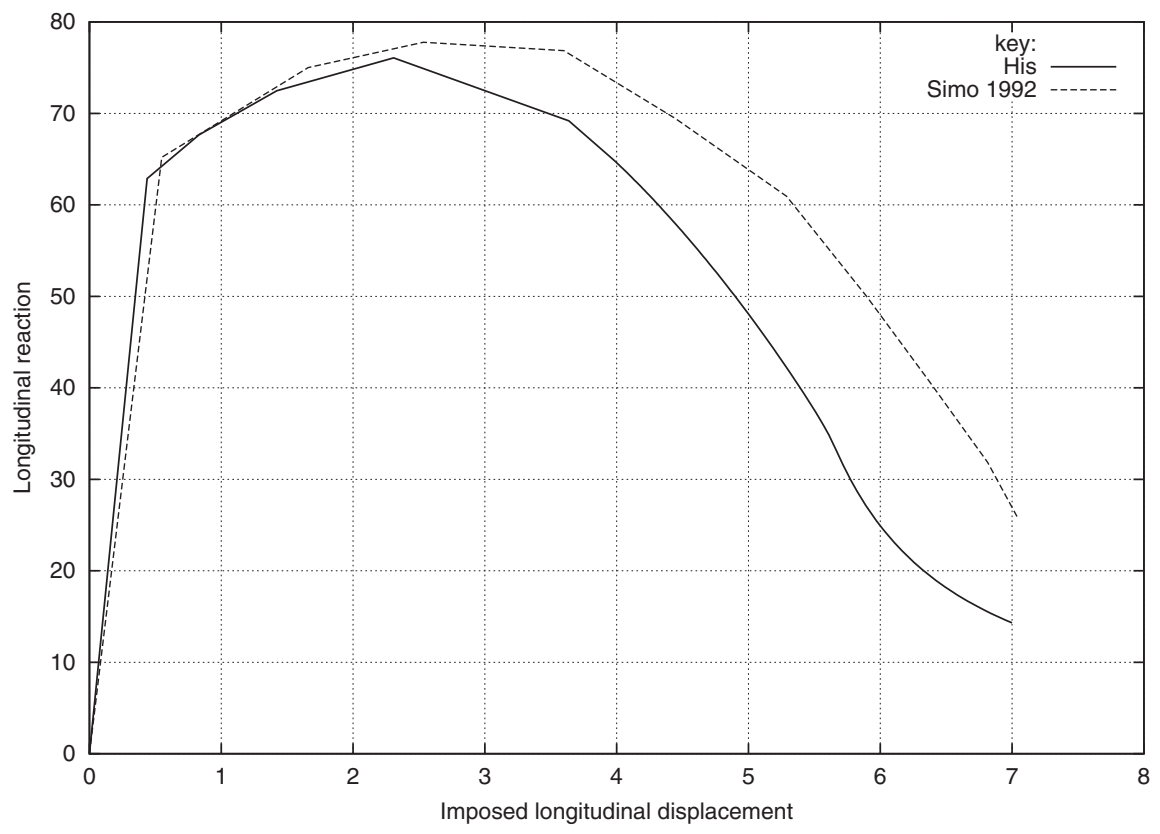

Figure 31. Load-displacement evolution for the tension test. Comparison with the results from Reference [18].
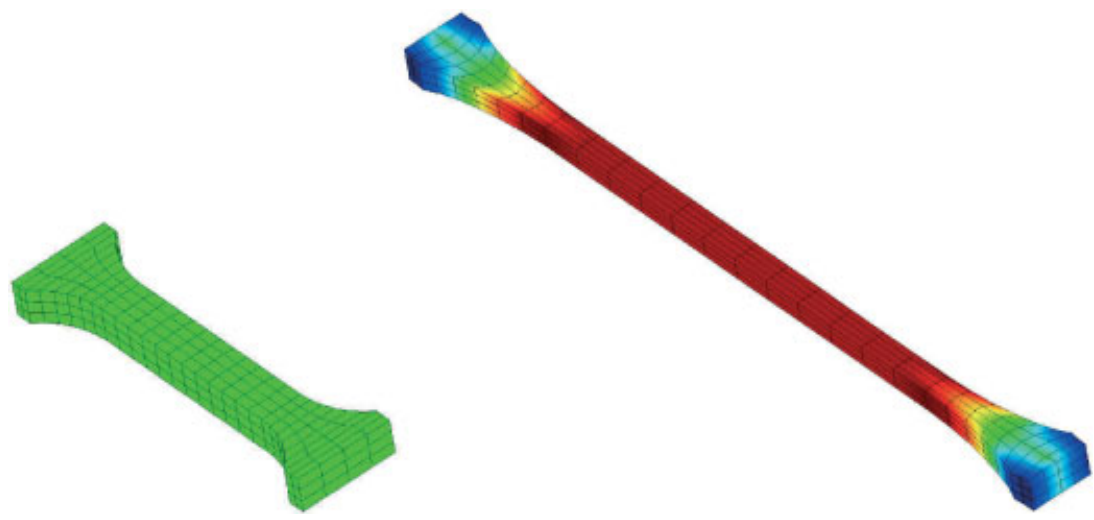

Figure 32. The deformed and non-deformed meshes for the silicone specimen. The longitudinal stress $\sigma_{L}$ contour plot is given over the deformed mesh. The red zones correspond to $\sigma_{L}=8.09$ and the blue zones to $\sigma_{L}=0.45$.

Further work with this formulation is being carried out, with the goal of reducing the number of internal variables. In addition, improvements are needed to attenuate mesh sensitivity of enhanced strain elements. 


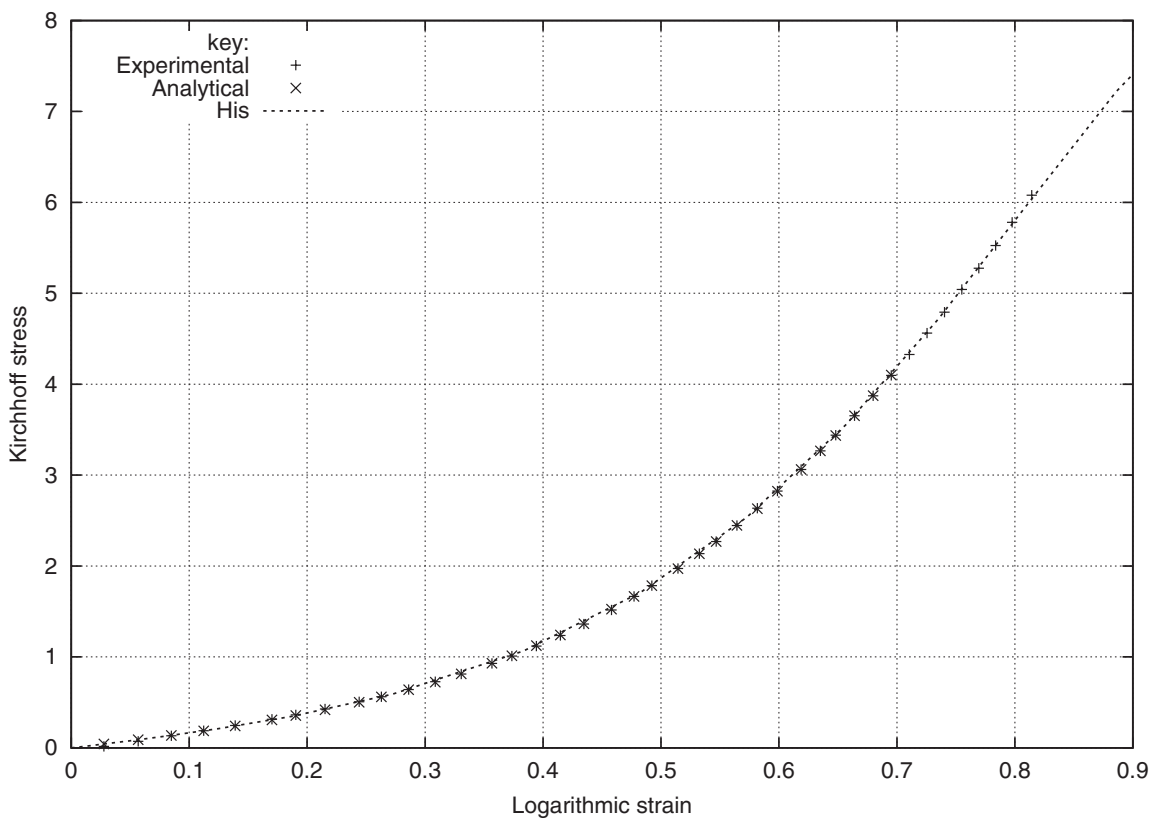

Figure 33. Stress-strain curve for the silicone specimen.

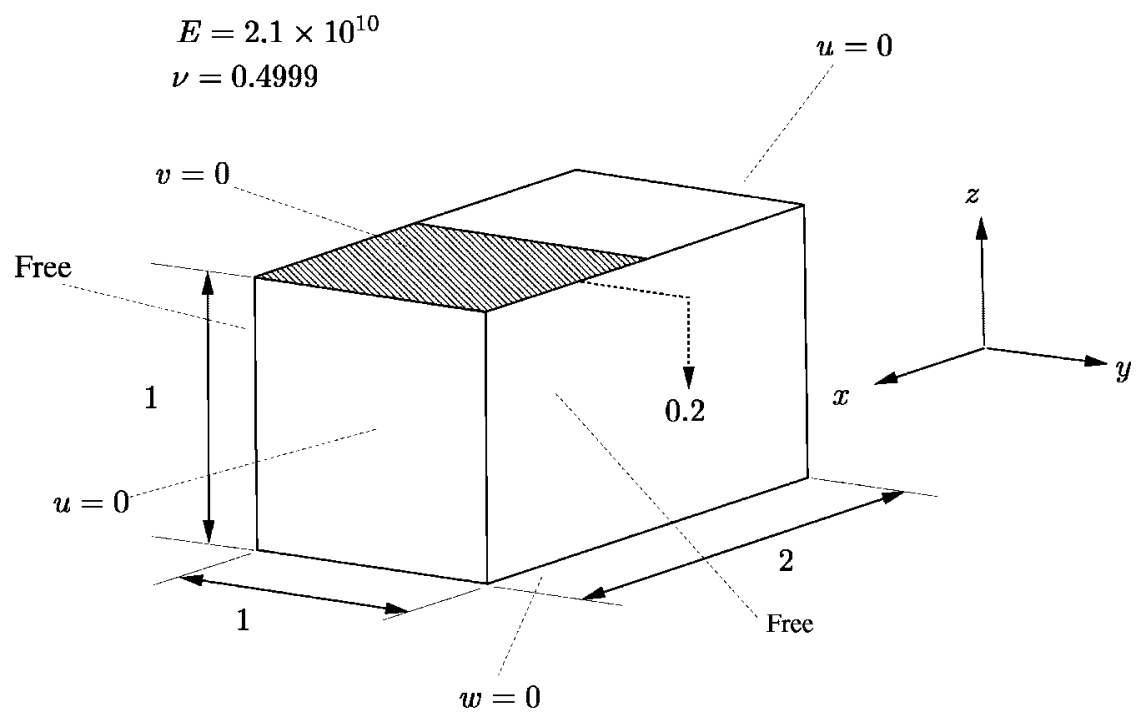

Figure 34. Indentation of a block: geometry and boundary conditions. 

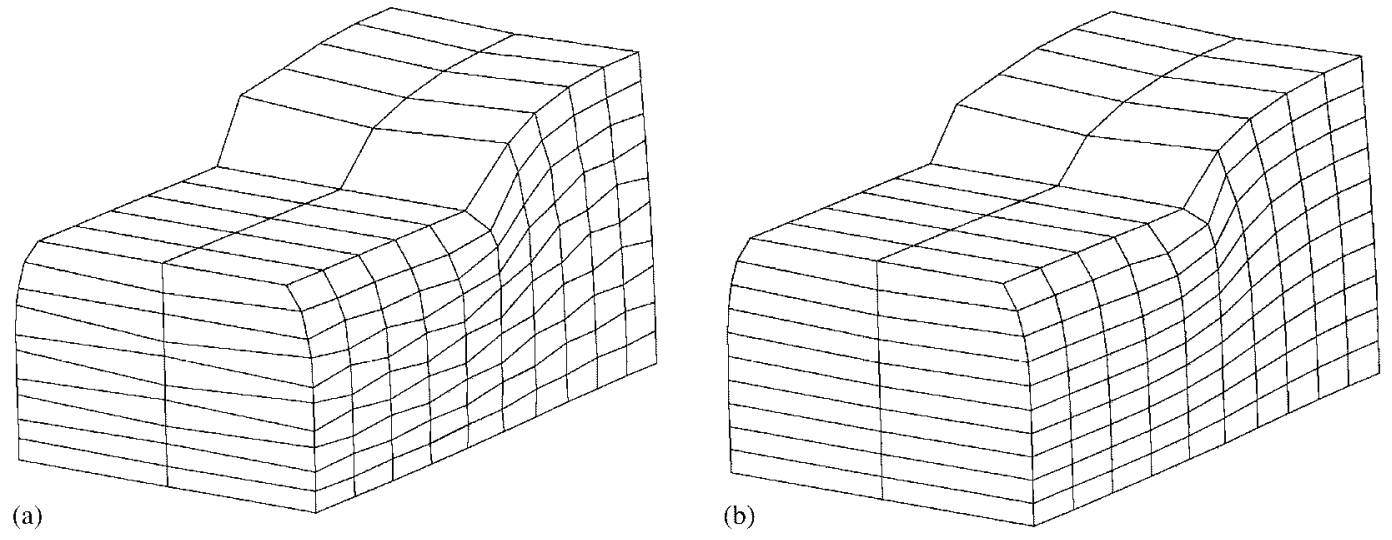

Figure 35. Indentation of a block: scaled $(10 \times)$ deformed mesh for $r=0$ and deformed mesh for the stabilized case $(r=1.5 E / 2(1+v)))$.

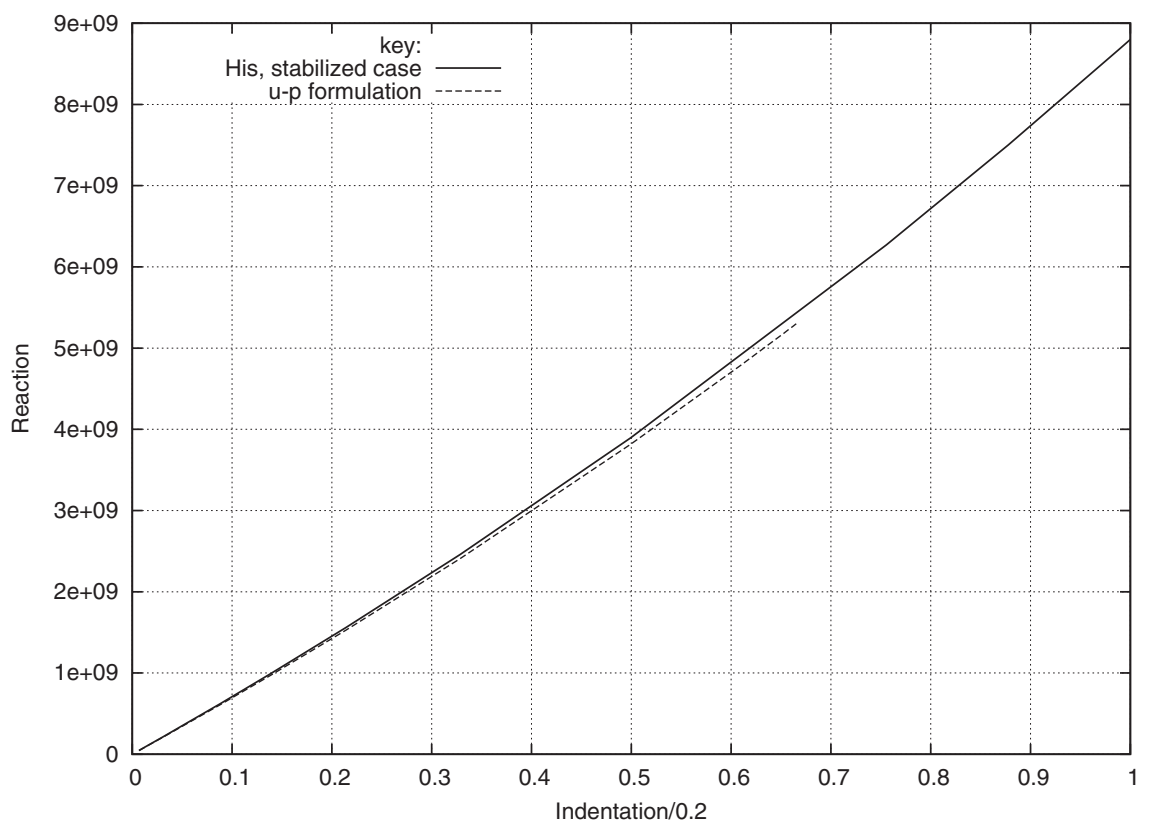

Figure 36. Indentation of a block: reaction curves for the stabilized case and comparison with a $u-p$ formulation.

\section{ACKNOWLEDGEMENTS}

This work was supported by 'Ministério da Ciência e da Tecnologia, FCT' under grant PRAXIS $\mathrm{XXI} / \mathrm{BD} / 18533 / 98$. This support is gratefully acknowledged. 
The experimental results for the silicone rubber tension test were provided by Professor António Torres Marques and his team in INEGI, Instituto de Engenharia Mecãnica e Gestăo Industrial. His expertise in the subject made this example possible.

\section{REFERENCES}

1. Sze KY, Ghali A. Hybrid hexahedral element for solids, plates, shells and beams by selective scaling. International Journal for Numerical Methods in Engineering 1993; 36:1519-1540.

2. Buechter N, Ramm E, Roehl D. Three-dimensional extension of non-linear shell formulation based on the enhanced assumed strain concept. International Journal for Numerical Methods in Engineering 1994; 37: 2551-2568.

3. Cho C, Park HC, Lee SW. Stability analysis using a geometrically nonlinear assumed strain solid shell element model. Finite Elements in Analysis and Design 1998; 29:121-135.

4. Hauptmann R, Schweizerhof K. A systematic development of solid-shell element formulations for linear and non-linear analyses employing only displacement degrees of freedom. International Journal for Numerical Methods in Engineering 1998; 42:49-69.

5. El-Abbasi N, Meguid SA. A new shell element accounting for through-thickness deformation. Computer Methods in Applied Mechanics and Engineering 2000; 189:841-862.

6. Hauptmann R, Doll S, Harnau M, Schweizerhof K. Solid-shell elements with linear and quadratic shape functions at large deformations with near incompressible materials. Computers and Structures 2001; 79:1671-1685.

7. Sze KY, Lo SH, Yao L-Q. Hybrid-stress solid elements for shell structures based upon a modified variational functional. International Journal for Numerical Methods in Engineering 2002; 53:2617-2642.

8. Andelfinger U, Ramm E. EAS-elements for two-dimensional, three-dimensional, plate and shell structures and their equivalence to HR-elements. International Journal for Numerical Methods in Engineering 1993; 36: 1311-1337.

9. Liu WK, Hu Y-K, Belytschko T. Multiple quadrature underintegrated finite elements. International Journal for Numerical Methods in Engineering 1994; 37:3263-3289.

10. Korelc J, Wriggers P. Efficient enhanced strain element formulation for 2D and 3D problems. In Advances in Finite Element Technology. Wiberg NE (ed.). CIMNE: Barcelona, Spain, 1995; 22-46.

11. Roehl D, Ramm E. Large elasto-plastic finite element analysis of solids and shells with the enhanced assumed strain concept. International Journal of Solids and Structures 1996; 33:3215-3237.

12. Weissman SL. High-accuracy low-order three-dimensional brick elements. International Journal for Numerical Methods in Engineering 1996; 39:2337-2361.

13. Chen Y-I, Stolarski HK. Extrapolated fields in the formulation of the assumed strain elements. Part II: Threedimensional problems. Computer Methods in Applied Mechanics and Engineering 1998; 154:1-29.

14. Liu WK, Guo Y, Belytschko T. A multiple-quadrature eight-node hexahedral finite element for large deformation elastoplastic analysis. Computer Methods in Applied Mechanics and Engineering 1998; 154:69-132.

15. Puso MA. A highly efficient enhanced assumed strain physically stabilized hexahedral element. International Journal for Numerical Methods in Engineering 2000; 49:1029-1064.

16. Taylor RL, Beresford PL, Wilson EL. A nonconforming element for stress analysis. International Journal for Numerical Methods in Engineering 1976; 10:1211-1219.

17. Simo JC, Rifai MS. A class of mixed assumed strain methods and the method of incompatible modes. International Journal for Numerical Methods in Engineering 1990; 29:1595-1638.

18. Simo JC, Armero F. Geometrically non-linear enhanced strain mixed methods and the method of incompatible modes. International Journal for Numerical Methods in Engineering 1992; 33:1413-1449.

19. Simo JC, Armero F, Taylor RL. Improved versions of assumed enhanced strain tri-linear elements for 3D finite deformation problems. Computer Methods in Applied Mechanics and Engineering 1993; 110:359-386.

20. Glaser S, Armero F. On the formulation of enhanced strain finite elements in finite deformations. Engineering Computations 1997; 14(7):759-791.

21. de Souza Neto EA, Peric D, Dutko M, Owen DRJ. Design of simple low order finite elements for large strain analysis of nearly incompressible solids. International Journal of Solids and Structures 1996; 33:3277-3296.

22. César de Sá JMA, Areias PMA, Natal Jorge RM. Quadrilateral elements for the solution of elasto-plastic finite strain problems. International Journal for Numerical Methods in Engineering 2001; 51:883-917.

23. Reese S, Kussner M, Reddy BD. A new stabilization technique for finite elements in non-linear elasticity. International Journal for Numerical Methods in Engineering 1999; 44:1617-1652.

24. Reese S, Wriggers P, Reddy BD. A new locking-free brick element technique for large deformation problems in elasticity. Computers and Structures 2000; 75:291-304.

25. Flanagan DP, Belytschko T. A uniform strain hexahedron and quadrilateral with orthogonal hourglass control. International Journal for Numerical Methods in Engineering 1981; 17:679-706. 
26. Belytschko T, Ong JS-J. Hourglass control in linear and nonlinear problems. Computer Methods in Applied Mechanics and Engineering 1984; 43:251-276.

27. Belytschko T, Bindeman LP. Assumed strain stabilization of the 4-node quadrilateral with 1-point quadrature for nonlinear problems. Computer Methods in Applied Mechanics and Engineering 1991; 88:311-340.

28. Bonet J, Bhargava P. A uniform deformation gradient hexahedron element with artificial hourglass control. International Journal for Numerical Methods in Engineering 1995; 38:2809-2828.

29. Hu Y-K, Nagy LI. A one-point quadrature eight-node brick element with hourglass control. Computers and Structures 1997; 65:893-902.

30. Li KP, Cescotto S. An 8-node brick element with mixed formulation for large deformation analyses. Computer Methods in Applied Mechanics and Engineering 1997; 141:157-204.

31. Pian THH, Sumihara K. Rational approach for assumed stress finite elements. International Journal for Numerical Methods in Engineering 1984; 20:1685-1695.

32. Sze KY, Yi S, Tay MH. An explicit hybrid stabilized eighteen-node solid element for thin shell analysis. International Journal for Numerical Methods in Engineering 1997; 40:1839-1856.

33. Sze KY, Sim YS, Soh AK. A hybrid stress quadrilateral shell element with full rotational D.O.F.S. International Journal for Numerical Methods in Engineering 1997; 40:1785-1800.

34. Piltner R, Taylor RL. A systematic construction of B-bar functions for linear and non-linear mixed-enhanced finite elements for plane elasticity problems. International Journal for Numerical Methods in Engineering 1999; 44:615-639.

35. Cao YP, Hu N, Lu N, Fukunaga H, Yao ZH. A 3D brick element based on hu-washizu variational principle for mesh distortion. International Journal for Numerical Methods in Engineering 2002; 53:2529-2548.

36. Bathe K-J, Dvorkin EN. A formulation of general shell elements-the use of mixed interpolation of tensorial components. International Journal for Numerical Methods in Engineering 1986; 22:697-722.

37. Bathe K-J. Finite Element Procedures. Prentice-Hall International Editions: Englewood cliffs, NJ, 1996.

38. Wagner W, Klinkel S, Gruttmann F. Elastic and plastic analysis of thin-walled structures using improved hexahedral elements. Computers and Structures 2002; 80:857-869.

39. Nagtegaal JC, Parks DM, Rice JR. On numerically accurate finite element solutions in the fully plastic range. Computer Methods in Applied Mechanics and Engineering 1974; 4:153-177.

40. Malkus DS, Hughes TJR. Mixed finite element methods-reduced and selective integration techniques: a unification of concepts. Computer Methods in Applied Mechanics and Engineering 1978; 15:63-81.

41. Hughes TJR. Generalization of selective integration procedures to anisotropic and nonlinear media. International Journal for Numerical Methods in Engineering 1980; 15(9):1413-1418.

42. Simo JC, Taylor RL, Pister KS. Variational and projection methods for the volume constraint in finite deformation elasto-plasticity. Computer Methods in Applied Mechanics and Engineering 1985; 51: $177-208$

43. Moran B, Ortiz M, Shih CF. Formulation of implicit finite element methods for multiplicative finite deformation plasticity. International Journal for Numerical Methods in Engineering 1990; 29:483-514.

44. Brink U, Stein E. On some mixed finite element methods for incompressible and nearly incompressible finite elasticity. Computational Mechanics 1996; 19:105-119.

45. Bonet J, Wood RD. Nonlinear Continuum Mechanics for Finite Element Analysis. Cambridge University Press: Cambridge, 1997.

46. Crisfield MA, Norris V. A stabilized large-strain elasto-plastic Q1/P0 method. International Journal for Numerical Methods in Engineering 1999; 46:579-592.

47. Holzapfel GA. Nonlinear Solid Mechanics: A Continuum Approach for Engineers. Wiley: New York, 2000.

48. Pantuso D, Bathe K-J. On the stability of mixed finite elements in large strain analysis of incompressible solids. Finite Elements in Analysis and Design 1997; 28:83-104.

49. Armero F. On the locking and stability of finite elements in finite deformations plane strain problems. Computers and Structures 2000; 75:261-290.

50. Kasper EP, Taylor RL. A mixed-enhanced strain method. Part I: Geometrically linear problems. Computers and Structures 2000; 75:237-250.

51. Areias PMA, César de Sá JMA, Conceição António CA. A gradient model for finite strain elastoplasticity coupled with damage. Finite Elements in Analysis and Design, 2003, in press.

52. Areias PMA, César de Sá JMA, Conceição António CA. Finite strain plasticity including softening: a gradient damage model. In Proceedings of the Fifth World Congress on Computational Mechanics (WCCM V), Mang HA, Rammerstorfer FG, Eberhardsteiner J (eds). July 7-12 2002. Vienna University of Technology, Austria. ISBN 3-9501554-0-6. http://wccm.tuwien.ac.at.

53. Ogden RW. Non-linear Elastic Deformations. Dover Publications: Mineola, New York, 1997.

54. Simo JC. Algorithms for static and dynamic multiplicative plasticity that preserve the classical return mapping schemes of the infinitesimal theory. Computer Methods in Applied Mechanics and Engineering 1992; 99: 61-112.

55. Miehe C. A constitutive frame of elastoplasticity at large strains based on the notion of a plastic metric. International Journal of Solids and Structures 1998; 35:3859-3897. 
56. Ramm E. The Riks/Wempner approach — an extension of the displacement control method in nonlinear analyses. Recent Advances in Non-linear Computational Mechanics, Chapter 3. Pineridge Press Limited: Swansea, UK, 1982; 63-86.

57. MacNeal RH, Harder RL. A proposed standard set of problems to test finite element accuracy. Finite Elements in Analysis and Design 1985; 1:1-20.

58. Irons B, Ahmad S. Techniques of Finite Elements. Ellis Horwood: Chichester, UK, 1980.

59. Timoshenko S, Woinowsky-Krieger S. Theory of Plates and Shells. (2nd edn). McGraw-Hill: New York, 1959.

60. Sansour C, Bocko J. On hybrid stress, hybrid strain and enhanced strain finite element formulations for a geometrically exact shell theory with drilling degrees of freedom. International Journal for Numerical Methods in Engineering 1998; 43:175-192.

61. Bucalem ML, Bathe K-J. Higher-order MITC general shell elements. International Journal for Numerical Methods in Engineering 1993; 36:3729-3754.

62. Betsch P, Stein E. Numerical implementation of multiplicative elasto-plasticity into assumed strain elements with application to shells at large strains. Computer Methods in Applied Mechanics and Engineering 1999; 179: $215-245$.

63. Bathe K-J, Iosilevich A, Chapelle D. An evaluation of the MITC shell elements. Computers and Structures 2000; 75:1-30.

64. Love AEH. A Treatise on the Mathematical Theory of Elasticity. Dover Publications: New York, 1996.

65. Betsch P, Menzel A, Stein E. On parametrization of finite rotations in computational mechanics. A classification of concepts with application to smooth shells. Computer Methods in Applied Mechanics and Engineering 1998; 155:273-305.

66. Massin P, Al Mikdad M. Nine node and seven node thick shell elements with large displacements and rotations. Computers and Structures 2002; 80:835-847.

67. Basar Y, Ding Y. Finite rotation shell elements for the analysis of finite rotation shell problems. International Journal for Numerical Methods in Engineering 1992; 34:165-169.

68. Buechter N, Ramm E. Shell theory versus degeneration - a comparison in large rotation finite element analysis. International Journal for Numerical Methods in Engineering 1992; 34:39-59.

69. Li M, Zhan F. The finite deformation theory for beam, plate and shell. Part 5. The shell element with drilling degree of freedom based on biot strain. Computer Methods in Applied Mechanics and Engineering 2000; 189:743-759.

70. Sansour C, Kollmann FG. Families of 4-node and 9-node finite elements for a finite deformation shell theory. an assessment of hybrid stress, hybrid strain and enhanced strain elements. Computational Mechanics 2000; 24: $435-447$.

71. Sabir AB, Lock AC. The application of finite elements to the large deflection geometrically nonlinear behaviour of cylindrical shells. In Variational Methods in Engineering, University Press: Southampton, 1972; $7 / 66-7 / 75$

72. Oliver J, Oñate E. A total lagrangian formulation for geometrically nonlinear analysis of structures using finite elements. Part I. two dimensional problems: shell and plate structures. International Journal for Numerical Methods in Engineering 1984; 20:2253-2281.

73. Sansour C, Bufler H. An exact finite rotation shell theory, its mixed variational formulation and its finite element implementation. International Journal for Numerical Methods in Engineering 1992; 34:73-115.

74. Hibbitt, Karlsson, Sorensen. Abaqus/Standard Example Problems Manual, 5.7, vol. I and II, 1997.

75. Arregui I, Destuynder P, Salaun M. An eulerian approach for large displacements of thin shells including geometric non-linearities. Computer Methods in Applied Mechanics and Engineering 1997; 140:361-381.

76. Eriksson A, Pacoste C. Element formulation and numerical techniques for stability problems in shells. Computer Methods in Applied Mechanics and Engineering 2002; 191:3775-3810.

77. Masud A, Tham CL, Liu WK. A stabilized 3D co-rotational formulation for geometrically nonlinear analysis of multi-layered composite shells. Computational Mechanics 2000; 26:1-12.

78. Jiang L. Chernuka MW. A simple four node co-rotational shell element for arbitrary large rotations. Computers and Structures 1994; 53:1123-1132.

79. Parish H. A continuum-based shell theory for non-linear applications. International Journal for Numerical Methods in Engineering 1995; 38:1855-1883.

80. Bischoff M, Ramm E. Shear deformable shell elements for large strains and rotations. International Journal for Numerical Methods in Engineering 1997; 40:4427-4449.

81. Wang L, Thierauf G. Finite rotations in non-linear analysis of elastic shells. Computers and Structures 2001 79:2357-2367.

82. Simo JC, Kennedy JG. On a stress resultant geometrically exact shell model. Part V nonlinear plasticity: Formulation and integration algorithms. Computer Methods in Applied Mechanics and Engineering 1992; 96:133-171. 
83. Brank B, Perić D, Damjanić FB. On large deformations of thin elasto-plastic shells: implementation of a finite rotation model for quadrilateral shell element. International Journal for Numerical Methods in Engineering 1997; 40:689-726.

84. Eberlein R, Wriggers P. Finite element concepts for finite elastoplastic strains and isotropic stress response in shells: Theoretical and computational analysis. Computer Methods in Applied Mechanics and Engineering $1999 ; 171: 243-279$.

85. Harnau M, Schweizerhof K. About linear and quadratic solid-shell elements at large deformations. Computers and Structures 2002; 80:805-817.

86. Korelc J, Wriggers P. Consistent gradient formulation for a stable enhanced strain method for large deformation. Engineering Computations 1996; 13(1):103-123. 